\title{
Analysis of Small-Scale Convective Dynamics in a Crown Fire Using Infrared Video Camera Imagery
}

\author{
Terry L. Clark, Larry Radke, Janice Coen, and Don Middleton \\ National Center for Atmospheric Research, Boulder, Colorado
}

(Manuscript received 23 June 1998, in final form 10 December 1998)

\begin{abstract}
A good physical understanding of the initiation, propagation, and spread of crown fires remains an elusive goal for fire researchers. Although some data exist that describe the fire spread rate and some qualitative aspects of wildfire behavior, none have revealed the very small timescales and spatial scales in the convective processes that may play a key role in determining both the details and the rate of fire spread. Here such a dataset is derived using data from a prescribed burn during the International Crown Fire Modelling Experiment. A gradient-based image flow analysis scheme is presented and applied to a sequence of high-frequency $(0.03 \mathrm{~s})$, high-resolution $(0.05-0.16 \mathrm{~m})$ radiant temperature images obtained by an Inframetrics ThermaCAM instrument during an intense crown fire to derive wind fields and sensible heat flux. It was found that the motions during the crown fire had energy-containing scales on the order of meters with timescales of fractions of a second. Estimates of maximum vertical heat fluxes ranged between 0.6 and $3 \mathrm{MW} \mathrm{m}^{-2}$ over the 4.5 -min burn, with early time periods showing surprisingly large fluxes of $3 \mathrm{MW} \mathrm{m}{ }^{-2}$. Statistically determined velocity extremes, using five standard deviations from the mean, suggest that updrafts between 10 and $30 \mathrm{~m} \mathrm{~s}^{-1}$, downdrafts between -10 and $-20 \mathrm{~m} \mathrm{~s}^{-1}$, and horizontal motions between 5 and $15 \mathrm{~m} \mathrm{~s}^{-1}$ frequently occurred throughout the fire.

The image flow analyses indicated a number of physical mechanisms that contribute to the fire spread rate, such as the enhanced tilting of horizontal vortices leading to counterrotating convective towers with estimated vertical vorticities of 4 to $10 \mathrm{~s}^{-1}$ rotating such that air between the towers blew in the direction of fire spread at canopy height and below. The IR imagery and flow analysis also repeatedly showed regions of thermal saturation (infrared temperature $>750^{\circ} \mathrm{C}$ ), rising through the convection. These regions represent turbulent bursts or hairpin vortices resulting again from vortex tilting but in the sense that the tilted vortices come together to form the hairpin shape. As the vortices rise and come closer together their combined motion results in the vortex tilting forward at a relatively sharp angle, giving a hairpin shape. The development of these hairpin vortices over a range of scales may represent an important mechanism through which convection contributes to the fire spread.

A major problem with the IR data analysis is understanding fully what it is that the camera is sampling, in order physically to interpret the data. The results indicate that because of the large amount of after-burning incandescent soot associated with the crown fire, the camera was viewing only a shallow depth into the flame front, and variabilities in the distribution of hot soot particles provide the structures necessary to derive image flow fields. The coherency of the derived horizontal velocities support this view because if the IR camera were seeing deep into or through the flame front, then the effect of the ubiquitous vertical rotations almost certainly would result in random and incoherent estimates for the horizontal flow fields. Animations of the analyzed imagery showed a remarkable level of consistency in both horizontal and vertical velocity flow structures from frame to frame in support of this interpretation. The fact that the 2D image represents a distorted surface also must be taken into account when interpreting the data.

Suggestions for further field experimentation, software development, and testing are discussed in the conclusions. These suggestions may further understanding on this topic and increase the utility of this type of analysis to wildfire research.
\end{abstract}

\section{Introduction}

High-intensity crown fires account for a significant percentage of the forest biomass burned throughout the world. Crown fires are forest fires that consume the

Corresponding author address: Dr. Terry L. Clark, National Center for Atmospheric Research, P.O. Box 3000, Boulder, CO 80307-3000. E-mail: clark@ncar.ucar.edu canopies of trees and (particularly when accompanied by other indicators of extreme fire behavior such as lofting of burning embers, intense burning, and rapid spread rates) can present a great hazard to firefighters. However, a good physical understanding of the initiation, propagation, and spread of crowning fires remains an elusive goal for fire researchers. Fire managers throughout the world continue to use empirically based fire-spread models in their fire-behavior prediction systems. Examples of such models are those derived from 
the work of McArthur in Australia (Nobel et al. 1980) and Rothermel (1972) in the United States. The essence of these models is that local fire spread rate is prescribed as a function of wind speed, terrain slope, humidity, and fuel characteristics. No account is taken, except for tuning the coefficients, for direct interactions between the fire and the atmosphere. Intense crown fires are an area where strong and nonlinear fire-atmosphere interactions occur and, therefore, stress the credibility of the empirical fire-spread models (Alexander et al. 1998). Further, the resulting unexpectedly violent fire behavior is a serious threat to the lives of firefighters and to plans for wildfire containment.

Recent increases in computer power make it possible for researchers to develop more physically based firespread models. Some notable attempts in this direction are the coupled fire-atmosphere simulations of Grishin (1992) and Clark et al. (1996a,b). Eventually this type of fully interactive model should allow realistic predictions of fire spread by explicitly treating the dominant physical processes. They also should allow researchers to understand better the range of fire-atmosphere interactions as well as the conditions under which they can be expected to occur. Such models then can be used to improve our understanding of fire behavior and to help to improve the operational fire-spread models used for actual fire spread and behavior predictions.

Fire spread involves the physics of convection, radiation, and mechanical advection effects such as those involved in rolling embers and spotting. The coupling of these processes combined with the physics and chemistry of combustion compose the essence of a fully coupled fire model. Such models already exist for pool fires (Tieszen et al. 1996) that involve the rupture of liquidhydrocarbon fuel tanks and subsequent ignition, where the combustion process is treated as diffusion limited. In forest or grass fires, the fuel is attached to the surface and is relatively porous, allowing air to blow through and around the fuel. This difference is seemingly minor but is important and can make forest and grass fires reaction-rate rather than diffusion limited, that is, limited by the time it takes fuel to burn given an ample supply of oxygen. As fire size increases, however, portions of the fire often are oxygen limited (Radke et al. 1991).

Intense vortices of various strengths and sizes often are observed in fires. These vortices may be created directly by the fire dynamics at the fire front or may involve interactions with atmospheric convection. Strong rotation was observed experimentally by Church et al. (1980) and in model simulations (e.g., Heilman 1992; Heilman and Fast 1992; Clark et al. 1996a,b). McRae and Flannigan (1990) describe fire vortices that in one case were observed to rip out and loft standing trees. One of us (Radke) observed a fire vortex rising more than $3 \mathrm{~km}$ above ground level (AGL), with flaming materials that were visible at about $2 \mathrm{~km}$. McGrattan et al. (1996) were successful in simulating a pool fire where counterrotating vortices were observed in the structure of the rising smoke. Banta et al. (1992) observed similar vortices in the Batersby and Left Hand Canyon fires using a $10-\mu \mathrm{m}$ lidar and a $3-\mathrm{mm}$ radar; the scale of the observed vortices was on the order of $100 \mathrm{~m}$. On the other hand, fire vortices on the scale of meters continually occur at the fire front, are an essential component of the convection, and almost certainly play a fundamental role in the physics of fire spread. Thus, vortex tilting not only is suspected of being a hazard in itself to nearby firefighters, who have described the formation of dangerous fire whirls and how they sometimes topple unpredictably to the ground, but is an important fire spread mechanism through both local dynamics and the ability to loft flaming objects into areas well removed from the former fire front.

One major difficulty in developing a realistic forest and grass fire model is field validation. Some data exist that describe rate of spread and some qualitative aspects of fire behavior; however, no wildfire data presently exist to describe the very small timescale and spatial scale involved in the convective processes that help to determine fire spread. This type of data is necessary for both physical guidance and the eventual validation of fully coupled fire-atmosphere biomass fire models.

The purpose of this paper is to attempt to derive such a dataset, albeit with some limitations, using data from the International Crown Fire Modelling Experiment (ICFME) (Alexander et al. 1998). A gradient-based image flow analysis scheme is presented and applied to a sequence of high-frequency, high-resolution radiant temperature images $(3-5 \mu \mathrm{m})$ obtained by an Inframetrics ThermaCAM instrument during an intense crown fire to derive wind fields and sensible heat flux. The present IR data were obtained using pixel scales that at the distances of $239-82 \mathrm{~m}$ correspond to resolutions of $0.16-0.05 \mathrm{~m}$, respectively, with a time resolution of $0.03 \mathrm{~s}$ per frame. This temporal resolution is much finer than currently is attained using either scanning radar or lidar and is necessary to resolve the very fine scales of motion that determine fire behavior. This data and analysis technique was used here to examine these very fine scales of motion to find energy-containing scales on the order of meters, with timescales of fractions of a second.

It is important to consider what the IR imagery represents in order to interpret physically the thermal and derived-velocity fields. First, it is clear that the IR image represents a sheet of data in the $x-z$ plane with the $y$ coordinate (depth) varying considerably over the image. As a result, apparent motions in the image may contain motions into or out of the image whereas we estimate only those that project onto the two-dimensional distorted sheet. For example, thermals that are estimated to be rising rapidly also may tilt and propagate toward the instrument as the air rises, that is, they may form turbulent bursts or hairpin vortices. The current dataset and analysis software are inadequate to allow quanti- 
tative estimation of these forward motions. Second, the depth of the distorted sheet of data is important. If the IR camera temperature represents an average over a significant depth into the flame front, then the IR temperatures and derived-velocity components are representative for similar depths. Both the coherency of the horizontal motions and the large regions of relatively cool IR temperatures $\left(<750^{\circ} \mathrm{C}\right)$ suggest that the high concentrations of soot expected in a crown fire such as this have limited the averaging depth to a shallow layer. It is the variability in distributions of hot soot and their motion that allows estimation of the fire winds. There is no way of estimating the maximum IR temperatures that might have existed in the saturated (i.e., radiant temperatures above $750^{\circ} \mathrm{C}$, the maximum that the ThermaCAM was recording) regions but they certainly were well above $750^{\circ} \mathrm{C}$. Maximum temperatures expected in such diffusion-type combustion are about $1200^{\circ} \mathrm{C}$. Further experimentation that combines observations of both the IR temperature and $\mathrm{CH}$ or $\mathrm{C}_{2}$ radicals, which occur only in the combustion zone, may help to resolve these issues.

\section{Background and motivation}

\section{a. Relationship between convection and radiation in wildfires}

A central question in forestry is the relative roles of radiative and convective heating in causing fire spread. Baines (1990) defined a dimensionless parameter $P$ as the ratio of radiative heating to convective cooling to assess whether radiation from the flame can cause fire propagation. Using idealized wind and flame structures, he argued that radiation from the flame cannot propagate the fire and that convection dominates when $P$ is less than 1 but that radiation from the flame probably can propagate the fire when $P$ is greater than 1, although this condition was not precise because he had omitted fuel-bed radiation. His particular model predicted that $P$ would increase with increasing fire size, with convection dominating small laboratory fires $(P<1)$ and radiation dominating large fires $(P>1)$.

We question this type of analysis for a number of reasons. First, our observations show that the radiant temperatures are similar in both grass and crowning forest fires, that is, they are mostly below about $750^{\circ} \mathrm{C}$ with intermittent regions of saturation above these temperatures. Thus, we have seen no indication that bigger natural biomass fires are significantly hotter. Second, and probably more important, one should expect a strong Reynolds number dependence associated with the character of convection. The Reynolds number is defined as $\operatorname{Re}=U l / \nu$, where $U$ is a characteristic velocity, $l$ is a characteristic length scale, and $\nu$ is the kinematic viscosity; small Re indicates laminar flow; if Re is large, the flow generally will be turbulent. Small fires, such as laboratory fire tunnels, have small to moderate Re whereas the much larger biomass fires have a much larger Re, which likely can become asymptotically large and indicate fully developed turbulence. Whether the fire winds are laminar or turbulent, there is always a strong interdependence between convection and radiation because convection is the vehicle that controls the flame surfaces from which the fire radiates onto the dry fuel. In low-Re fires, one observes well-structured flow, whereas for large Re the local vorticity dynamics lead to the type of structures seen in highly turbulent flow. Instead of simply encountering hot air rising in wellorganized thermals, one encounters vortex tilting that leads to intense motions in almost every conceivable direction at the fire front. Our current observations and previous three-dimensional coupled fire modeling (Clark et al. 1996a,b) corroborate this point.

Since the manifestations of vorticity production and its resulting levels of turbulence scale with Re and thermal forcing, it is not clear at this time how one might attempt to scale the relative importance of radiation versus convection or whether this question is even well posed. Thus, it is clear that a good understanding of fire behavior demands a clear picture of the nature of the three-dimensional local fire wind, particularly at high Re when it is strongly turbulent. The relationship between radiation and convection logically will follow.

\section{b. Application of infrared cameras to fire research}

Sequences of visual camera images often have been used to calculate atmospheric motions in isolated smoke plumes (e.g., Templeman et al. 1990; Hidalgo 1993). With developments in IR technology, sequences of IR images could be analyzed to discern plumes from their environments more distinctly (Rickel et al. 1990), although the overwhelming amount of data provided by IR video cameras motivated users to limit their analyses to selected images every few minutes.

Infrared cameras have been used successfully to detect and map wildfires. Although human eyes are very sensitive receivers to radiation with wavelengths in the visible range between 0.4 and $0.7 \mu \mathrm{m}$, we are effectively blind to thermal emissions in the $3-15-\mu \mathrm{m}$ IR wavelengths. Because these thermal emissions are proportional to the fourth power of the surface temperature, they are largely independent of solar illumination, and so IR cameras are effective both at night and during the day.

Increasingly sensitive image converters are being made available now and we can think of them as television cameras, sensitive in the IR. This synthetic vision gives a familiar view of surroundings but offers some useful advantages to fire researchers. For example, very hot objects such as smoldering coals do not stand out in our vision but appear very bright in the IR.

Furthermore, IR vision provides a large comparative advantage over normal vision when the field of interest is obscured by smoke and the electromagnetic radiation 
is being scattered and absorbed by interactions with smoke aerosols. Smoke very effectively (on a mass basis) obscures vision because of very efficient electromagnetic interactions that result from the size of smoke particles. Biomass fires produce smoke particles that are mostly in the 0.1-1.0- $\mu \mathrm{m}$ sizes (Radke et al. 1991) that span the wavelengths of normal vision $(0.4-0.7 \mu \mathrm{m})$. Since attenuation peaks when the wavelengths and aerosol particles are the same "size" (Ditchburn 1963), the ability to observe through smoke is improved greatly by increasing the wavelength of the viewing imager. Our 3-5- $\mu \mathrm{m}$ imager can observe the hot flaming fire structures clearly when they are shrouded so completely by thick smoke that not a hint of flame can be discerned visually.

\section{Description of experiment and instrumentation}

In this work, the high-frequency, high-resolution passive measurements of infrared radiation in the $3-5-\mu \mathrm{m}$ range are analyzed to derive atmospheric wind velocities during the crown fire that occurred during the burning of plot 6 in ICFME.

\section{a. International Crown Fire Modelling Experiment}

The ICFME took place between 19 June and 12 July 1997, about $40 \mathrm{~km}$ northeast of Fort Providence in the Northwest Territories (NWT) of Canada. This area contains boreal forest composed primarily of an overstory of 12-m-tall 65-yr-old jack pine with a dense understory of black spruce. Isolated rectangular forest plots of 150 $\mathrm{m}$ per side were created and then instrumented for burns in weather conditions that favor the development of crowning fires. Instrumentation included an array of ground temperature sensors, towers of radiometers, automatic weather stations, and IR and visual cameras. Three plots were burned during the initial year of the experiments; the burn of plot 6 on 9 July 1997 was particularly successful. Analyses of IR data taken for this burn with an Inframetrics ThermaCAM SC1000 will be presented.

Figure 1 shows a schematic of the prepared forest plots. The cleared perimeters were $50 \mathrm{~m}$ wide. Numbers that identify the plots are shown along with the dates on which they were burned during the 1997 experiment. A cross in the clearing to the southeast of plot 6 marks the position of the U.S. Forest Service (USFS) 50-ft tower. The National Center for Atmospheric Research's (NCAR) IR camera plus two USFS visual cameras were positioned at the top of this tower. One visual camera was a regular Super Video Home System/National Television System Committee (SVHS/NTSC) camcorder; images from this burn were part of a USFS video (NWT Crown Fire Experiment, July 1997). The second visual camera was a USFS high-speed camera that took 15 seconds of film at 250 frames per second. A second cross approximately $150 \mathrm{~m}$ to the east of the southeast

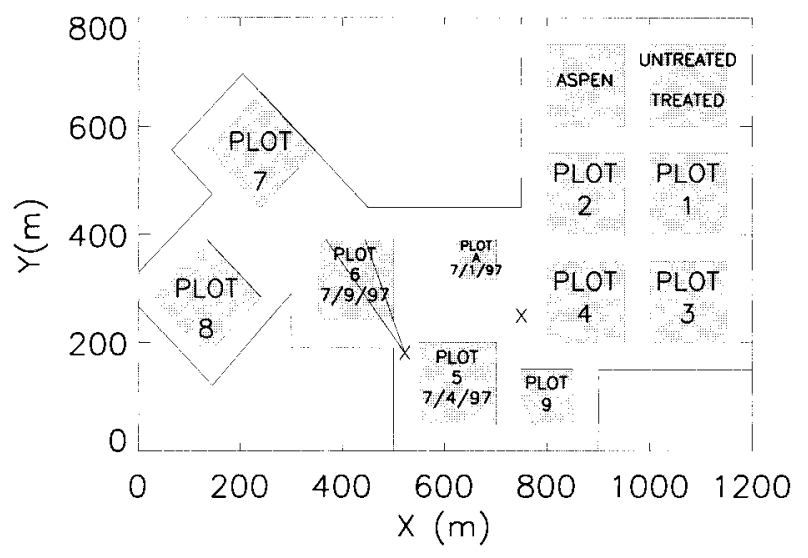

FIG. 1. Outline of burn plots for ICFME in Jun-Jul 1997. The forested areas are shaded. Areas between are the cleared fire breaks. The thin perimeter line marks the boundary of the experimental area, outside of which are trees and muskeg. The dates indicate when, if at all, the plot was burned during the 1997 experiment. The crosses mark locations of IR cameras and the two lines extending from the cross nearest plot 6 represent NCAR's IR camera's FOV (see text for further details). North is toward the top of the page.

corner of plot 6 marks the location of the Canadian Forest Service's (CFS) IR line scanner that also operated during this experiment. We have yet to analyze any of their data from this burn but we were able to view the data and will comment on it later. Their view from the side proved particularly revealing in regard to certain aspects of the dynamics.

\section{b. Meteorological conditions on 9 July 1997 and the plot-6 burn}

The three experimental crown fires conducted at ICFME in 1997 were, to our knowledge, the most well monitored of such experimental fires to date. Here, analyses of the burn of plot 6 are presented.

On 9 July 1997 the wind was between 16 and $17 \mathrm{~km}$ $\mathrm{h}^{-1}$ from the north to north-northeast during the burn. The temperature and relative humidity were $24^{\circ} \mathrm{C}$ and $45 \%$, respectively. The estimated total fuel load was 4.3 $\mathrm{kg} \mathrm{m}^{-2}$, while $1.9 \mathrm{~kg} \mathrm{~m}^{-2}$ of this load was estimated to contribute to the advancing flame front. The plot was ignited at the north face using a Terra Torch (a truckmounted pressurized flame thrower) held by a jogger on the ground behind a vehicle holding the liquid napalm. The ignition started at the northeast corner and proceeded to the northwest corner, taking approximately $50 \mathrm{~s}$. The fire crowned almost immediately and burned through to the southern end in approximately $4.5 \mathrm{~min}$, which converts to an average spread rate of about 0.6 $\mathrm{m} \mathrm{s}^{-1}$. Photographs from an overhead helicopter show that the fire took on a parabolic shape. The visual fire line width was estimated to be approximately $30 \mathrm{~m}$ during the burn (D. Latham 1998, personal communication). Evidence suggests that the fire was still accelerating at the conclusion of the burn, indicating that the 
maximum spread rate was considerably greater than the $0.6 \mathrm{~m} \mathrm{~s}^{-1}$.

The accelerating nature of the burn was evident in the outline of spotting in the break to the east of plot 6. Strong updrafts in the burning fuel result in countless fire brands filling the atmosphere near the fire. The fire brands are lofted in all directions and ignite nearly all exposed roots in the cleared regions between the plots that are within the envelope of the fire-brand trajectories. When the plot- 6 burn ended, a well-defined outline of burning roots remained, extending from the northeast corner of plot 6 and linearly expanding to the east of plot 6 for the entire length of the burn. We estimate the angle at which the spotting expanded with distance was approximately $6^{\circ}$. Our interpretation of the spotting envelope's linear increase with distance is that the convective motions in the fire were continually increasing, causing spotting to extend farther with time.

\section{c. Inframetrics ThermaCAM}

The Inframetrics ThermaCAM SC1000 (TCAM) is a palm-sized hand-held infrared focal plane array radiometer designed for scientific, research, and laboratory temperature measurements. The sensing array is cooled to cryogenic temperatures with a miniature Stirling thermodynamic cycle refrigerator, eliminating the need to cool with liquid nitrogen. Unlike scanning instruments, each element in a mosaic of $256 \times 256$ platinum silicide detectors stares continuously, providing a high thermal sensitivity. The TCAM, weighing $3.7 \mathrm{lb}(1.7 \mathrm{~kg})$, provides temperature measurement accuracy of better than $\pm 2^{\circ} \mathrm{C}$ at temperatures up to $2000^{\circ} \mathrm{C}$ and can resolve temperature differences of less than $0.1^{\circ} \mathrm{C}$. The temperature range is selected by choosing the temperature at the bottom of the range; in this work, the range was set to be $400^{\circ}-750^{\circ} \mathrm{C}$.

The camera has interchangeable lenses and filters, various remote control options, extensive postprocessing software, and battery operation. Each lens incorporates a system of thermal sensors and lens identification magnets for automatic recognition and calibration by the system. The use of an ambient temperature compensation program and LOWTRAN-7 atmospheric attenuation correction model built into the camera enables accurate, drift-free measurements. This system has benefits over other bulky infrared measuring devices, in that it does not require a large external cooling device or cryogenic liquids and can be held and directed like a visual video camera.

The two lines drawn between the USFS tower and plot 6 in Fig. 1 represent the field of view (FOV) of NCAR's IR camera during the burning of plot 6 . The average distances to the southern and northern edges of the plot's fuel were 63 and $239 \mathrm{~m}$, respectively. The camera images were recorded continuously during the burn using a standard SVHS/NTSC video recorder at 30 frames per second.

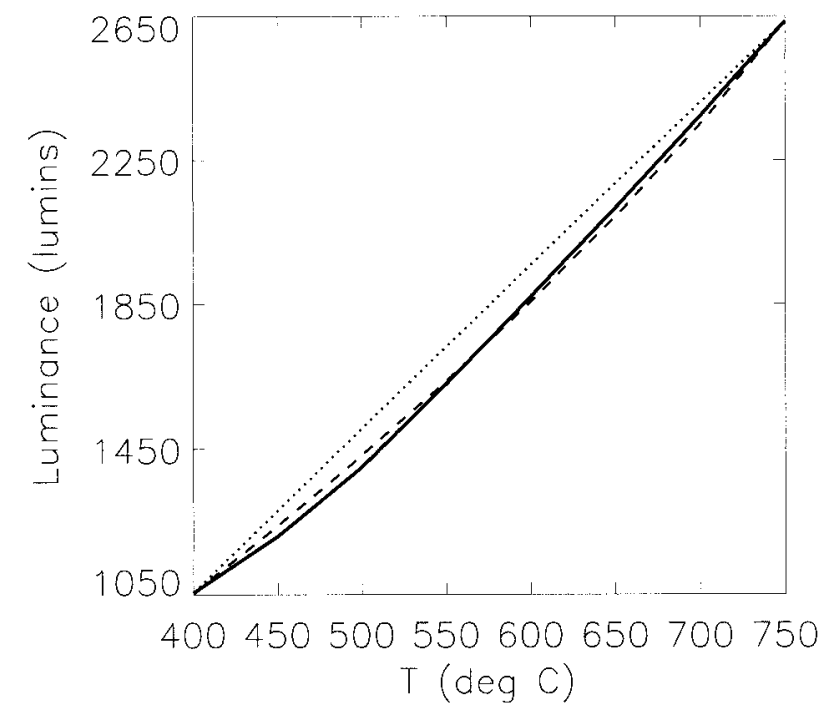

FIG. 2. Calibration curve used to convert luminance measured by the Inframetrics ThermaCAM SC1000 camera to infrared temperature. Solid, dashed, and dotted lines represent factory calibration, quadratic fit, and linear fit, respectively.

The analog information recorded on SVHS media was sampled into a motion-JPEG (Joint Photographic Experts Group standard for video) digital format using a quality factor of 0.75 , giving a separate disk file for every frame. Motion-JPEG is a lossy (some amount of unneeded data is lost during compression) intraframe (nontemporal) compression scheme that employs a discrete cosine transformation on $8 \times 8$ pixel regions. A transfer from analog into an uncompressed digital format would have been ideal but was not possible due to bandwidth limitations in the digitizing system. The motion-JPEG data subsequently were converted into a stream of 8100 uncompressed, byte-scaled grayscale images covering the 4.5-min burn.

After image conversion using motion-JPEG, $432 \times$ 478 pixels of digital data per frame were retained. For the $17^{\circ} \times 16^{\circ}$ Inframetrics lens, this resolution translates to pixels that span approximately $0.165 \mathrm{~m} \times 0.122 \mathrm{~m}$ at the beginning of the burn (looking at the distant flames) to $0.044 \mathrm{~m} \times 0.036 \mathrm{~m}$ (looking at the nearest flame front).

The process of converting digital camera data to analog and then back to digital using motion-JPEG introduces appreciable noise into the data. Furthermore, tape quality degrades severely with copying, and a copy was used to create the digital files. This noise is exacerbated further because the $60-\mathrm{Hz}$ rate of NTSC combines two scans to form a single frame. Thus, each frame is composed of two interlacing frames, the first recorded using only odd-numbered lines and the second with evennumbered lines. As a result, some of the noise is systematic.

Figure 2 shows the calibration curve used to convert luminance to IR temperature. The calibration curve is 
weakly nonlinear and is approximated easily using a polynomial fit. The factory-supplied calibration is shown by the solid line. We use a quadratic fit that is shown by the dashed line, and the straight dotted line demonstrates the degree of nonlinearity in the calibration. Our quadratic approximation for the IR temperature $f$ is

$$
f=400+1.728436 g-0.00139562 g^{2},
$$

where $g$ is the resulting 1-byte digitized datum representing the luminance. Values of $g$ of 0 and 255 represent luminance values of 1055 and $2636 \mathrm{~lm}$, respectively. This conversion uses a calibrated emissivity. Our calibration assumed that the emitter is a blackbody, since the emitters in these cases are mainly glowing soot particles, which have an emissivity close to 1.0.

The digitized data from the 4.5-min burn amount to 2.5 Gbytes. This amount was too large to process at once so the analysis was limited to 17 windows of data equally spaced $0.25 \mathrm{~min}$ apart. Sixteen consecutive frames of data were retained for each window except for a later one in which 100 consecutive frames were retained. Large segments of the 4.5 min were processed and rendered into video clips that are described at the end of section 5 .

\section{Description of image flow analysis}

\section{a. Image flow algorithm}

We considered gradient-based methods of image processing to be the most applicable for extracting wind estimates from the IR imagery. Horn and Schunck (1981) initially proposed using a single equation that describes the conservation of irradiance. Their method to formulate a unique solution was to cast the problem in a variational form by adding a smoothness constraint. This approach and subsequent developments for evaluating image motion are reviewed by Schunck (1986). Schunck also describes alternate methods such as difference picture, matching, and correlation. Tuttle and Foote (1990) use a correlation technique to derive boundary layer winds from single Doppler radar data. Verri et al. (1990) present more general methods to estimate two-dimensional motion in the image's plane. This approach involves describing the motion as a combination of the five elementary motions from Helmholtz's theorem for deformable objects. These motions are composed of two components of translation, a rotation, a uniform expansion, and two orthogonal components of shear. As an example, Bab-Hadiashar and Suter (1995) and Bab-Hadiashar et al. (1996) minimize a functional composed of four of the six elementary motions, namely, the two components of translation, rotation, and pure expansion.

Their method was considered to be too advanced for our first attempt to derive fire winds from IR imagery. Instead, we chose to solve just the two components of translation and used these results to assess whether to consider more complete image flow analysis in the future. In the following equations the subscripts generally indicate first- or second-order partial differentiation w.r.t. the subscript variable. The governing equation for image flow is

$$
\frac{d}{d t} f=f_{t}+u f_{x}+w f_{z}=0,
$$

where $f$ represents, in this case, the IR temperature $T_{\mathrm{IR}}$. The variables $u$ and $w$ are the horizontal ( $x$ direction) and vertical ( $z$ direction) components of the wind, respectively. Time is indicated by $t$. The basic assumption is that $f$ is approximately conserved over the short time required for analysis. Here, this would be $0.03-0.07 \mathrm{~s}$. If it is assumed that $u$ and $w$ are locally translational, then one can take the gradient of (2) to form the matrix equation

$$
\left(\begin{array}{ll}
f_{x x} & f_{x z} \\
f_{x z} & f_{z z}
\end{array}\right)\left(\begin{array}{l}
u \\
w
\end{array}\right)=\left(\begin{array}{l}
-f_{t x} \\
-f_{t z}
\end{array}\right),
$$

which describes pure translation. The determinant det of (3) is

$$
\operatorname{det}=f_{x x} f_{z z}-f_{x z}^{2} .
$$

Providing there is sufficient curvature to the flow, that is, |det| is relatively large, then well-conditioned solutions for $u$ and $w$ can be obtained. The possibility that (3) could be ill defined is termed the "aperture problem" (e.g., Verri et al. 1990).

Solutions of (3) for the fire imagery result in a fine mosaic formed by the contours of zero eigenvalues. One can obtain reasonable solutions for $u$ and $w$ in pixels located within most tiles of this mosaic that are well removed from any corner. Nagel (1983) gives a topological description of the grayscale field, from visual imagery, discusses the so-called corner problem, and presents solutions. Again, this approach is something that we may want to consider in the future. Instead we chose to solve (3) using a variation of the robust statistical and least mean squares approach used by BabHadiashar and Suter $(1995,1997)$. This approach is applied easily to our problem and is computationally efficient.

The image flow method consists of two steps. First (3) is solved to identify outliers. Whenever solutions to (3) give wind speeds greater than, say, $40 \mathrm{~m} \mathrm{~s}^{-1}$, that particular pixel is flagged as an outlier. Once outliers are identified, the solution of (3) is formulated in the least squares approach using an affine model, that is, one in which transformations such as translation, rotation, or uniform stretching map into polynomial fits. Variables $u$ and $w$ are locally approximated as $\hat{u}, \hat{w}$, where

$$
\hat{u}=a_{0}+a_{1} x+a_{2} z,
$$

and 


$$
\hat{w}=b_{0}+b_{1} x+b_{2} z
$$

The functional $\Lambda$ is formulated as

$$
\begin{aligned}
\Lambda=\sum_{i} \sum_{j} \nu_{i, j} & {\left[\left(\hat{u} f_{x x}+\hat{w} f_{x z}+f_{x t}\right)^{2}\right.} \\
& \left.+\left(\hat{u} f_{x z}+\hat{w} f_{z z}+f_{z t}\right)^{2}\right]
\end{aligned}
$$

and minimized to form the symmetric sixth-order matrix equation [using a $2 \mathrm{~d}$-order fit in (5) and (6) results in 12 th-order matrices]:

$$
\left(\begin{array}{ll}
\mathbf{A} & \mathbf{C} \\
\mathbf{C} & \mathbf{B}
\end{array}\right)\left(\begin{array}{l}
\mathbf{a} \\
\mathbf{b}
\end{array}\right)=\left(\begin{array}{l}
\mathbf{g}_{1} \\
\mathbf{g}_{2}
\end{array}\right) .
$$

Here $\nu_{i, j}$ is normally taken as unity except for outlier points where $\nu_{i, j}=0$. The summation in (7) is usually taken over a $7 \times 7$ local patch of pixels.

Using the definitions

$$
\begin{aligned}
& \mathrm{am}=f_{x x}^{2}+f_{x z}^{2}, \\
& \mathrm{bm}=f_{z z}^{2}+f_{x z}^{2}, \\
& \mathrm{~cm}=f_{x z}\left(f_{x x}+f_{z z}\right), \\
& g 1=-f_{x t} f_{x x}-f_{z t} f_{x z},
\end{aligned}
$$

and

$$
g 2=-f_{x t} f_{x z}-f_{z t} f_{z z},
$$

we can write (where summations here and subsequently are over a local path of data and dot products are used) A as

$$
\mathbf{A}=\left(\begin{array}{ccc}
\sum \text { am } & \sum x \cdot \text { am } & \sum z \cdot \mathrm{am} \\
\sum x \cdot \mathrm{am} & \sum x^{2} \cdot \mathrm{am} & \sum x z \cdot \mathrm{am} \\
\sum z \cdot \mathrm{am} & \sum x z \cdot \mathrm{am} & \sum z^{2} \cdot \mathrm{am}
\end{array}\right)
$$

and similarly for $\mathbf{B}$ and $\mathbf{C}$ by replacing am with bm and $\mathrm{cm}$, respectively. Here $\mathbf{a}^{\mathrm{T}}=\left[a_{0}, a_{1}, a_{2}\right]$ and $\mathbf{b}^{\mathrm{T}}=\left[b_{0}\right.$, $\left.b_{1}, b_{2}\right]$, respectively, and the transposes of the righthand side components of (8) are

$$
\mathbf{g}_{1}^{\mathrm{T}}=\left[\sum g 1, \sum x \cdot g 1, \sum z \cdot g 1\right]
$$

and

$$
\mathbf{g}_{2}^{\mathrm{T}}=\left[\sum g 2, \sum x \cdot g 2, \sum z \cdot g 2\right] .
$$

Here (8) reduces to the simplest equation set when the first-order polynomial is reduced to zeroth order, that is, for a fixed $u$ and $w\left(a_{1}=a_{2}=b_{1}=b_{2}=0\right)$. In this case, the normal matrix equation is obtained:

$$
\left(\begin{array}{cc}
\sum \mathrm{am} & \sum \mathrm{cm} \\
\sum \mathrm{cm} & \sum \mathrm{bm}
\end{array}\right)\left(\begin{array}{l}
u \\
w
\end{array}\right)=\left(\begin{array}{c}
\sum g 1 \\
\sum g 2
\end{array}\right) .
$$

One might consider using (17) because it is far more efficient than (8). As will be shown, (17) worked reasonably well in kinematic tests; however, this method did not work well for the actual fire winds because of the extreme gradients and highly transient nature of the flow.

Whether (8) or (17) are used to estimate local winds, rejection criteria that will adequately identify unacceptable solution points are required. The method adopted is to test the innermost matrix inverse(s). In solving (8), the inverse matrix is calculated as

$$
\left(\begin{array}{cc}
\mathbf{F}^{-1} \mathbf{A}^{-1} & -\mathbf{F}^{-1} \mathbf{A}^{-1} \mathbf{C} \mathbf{B}^{-1} \\
-\mathbf{G}^{-1} \mathbf{B}^{-1} \mathbf{C} \mathbf{A}^{-1} & \mathbf{G}^{-1} \mathbf{B}^{-1}
\end{array}\right),
$$

where

$$
\mathbf{F}=\mathbf{I}-\mathbf{A}^{-1} \mathbf{C B}^{-1} \mathbf{C}
$$

and

$$
\mathbf{G}=\mathbf{I}-\mathbf{B}^{-1} \mathbf{C A}^{-1} \mathbf{C} .
$$

In this case, data are rejected if either

$$
\sum \mid \mathbf{F}^{-1} \mathbf{F}-\|^{2}>r_{f}^{2}
$$

or

$$
\sum \mid \mathbf{G}^{-1} \mathbf{G}-\|^{2}>r_{g}^{2},
$$

where some finite value for variance $r_{f}^{2}$ and $r_{g}^{2}$, say, $\approx 0.1$, is chosen. The purpose of (21) and (22) is automatically to identify rejected points dominated by outliers. In such cases, the determinants of $\mathbf{A}$ and $\mathbf{B}$ already will have been given nonzero default values to avoid division by zero. The threshold velocity used in the initial outlier identification can be reasonably large to optimize the inclusion of most of the available data. The equivalent criteria to (21) are used in the solution of (17). A final outlier calculation is performed in which solutions of $u$ and $w$ are rejected if either component is larger than, say, $40 \mathrm{~m} \mathrm{~s}^{-1}$. No outliers were encountered after solving (8); however, outliers were found after solving (17) for the fire winds.

\section{b. Numerical approximations and filters}

Estimating velocities using gradient-based methods requires that spatial and temporal derivatives be evaluated. Since gigabytes of data existed, computationally efficient methods were sought. In addition, the data may contain considerable noise.

To process the data, first a 1-2-1 temporal filter is applied a selected number of times as

$$
f_{t}^{\tau+1}=\left(f_{t-\Delta t}^{\tau}+2 f_{t}^{\tau}+f_{t+\Delta t}^{\tau}\right) / 4,
$$

where $\tau$ denotes an iteration index. The number of time levels required to filter the data temporally is $3+2 \tau$; that is, one application $(\tau=1)$ requires five time levels. Successive passes through the 1-2-1 temporal filter, always using equally filtered data, were applied until two or three time levels of data remained. These remaining data were used to calculate the time derivative and mid-time-level $(t+\Delta t / 2)$ fields. The first time 
derivative $f_{t}$ is determined using either the centered approximation,

$$
f_{t}=\frac{f(t+\Delta t)-f(t-\Delta t)}{2 \Delta t},
$$

or the forward-in-time approximation,

$$
f_{t}=\frac{f(t+\Delta t)-f(t)}{\Delta t} .
$$

In the case of (24), $f(t)$ is used in the remaining calculations of spatial derivatives whereas for (25) $\bar{f}$ is used, where $\bar{f}=[f(t+\Delta t)+f(t)] / 2$. While (25) provides better time resolution to the data it also tends to produce somewhat noisier solutions than (24). We are still assessing advantages of (24) over (25) for the IR fire data.

After the data have been filtered temporally and the first time derivative has been calculated, one is left with fields of $f$ and $f_{t}$ at each pixel. The next step is to reduce spatial noise in these fields by applying a Gaussian filter. A symmetrical Gaussian of the form

$$
G(x, z)=c^{*} \exp \left[-\frac{\left(x^{2}+z^{2}\right)}{\sigma^{2}}\right]
$$

is convolved over the data. The leading coefficient in (26), $c^{*}[\approx 1 /(2 \pi \sigma)]$, is calculated to ensure that the numerically approximated weights add to unity. The value of $\sigma$ was determined through tuning experiments. The typical value chosen gave a weighting ratio of 1.4 between neighboring pixels on either the $x$ or $z$ axis. The area over which the Gaussian is convolved over the fields ranges from $5 \times 5$ to $9 \times 9$ pixels, that is, an area of order 5-9. The number of points used (order of area) and the neighbor-weighting ratios are noted in the analyzed figures.

The method adopted for estimating spatial derivatives is to use a local least squares polynomial fit. The variable $\hat{f}$ is defined as

$$
\hat{f}=c_{0}+c_{1} x+c_{2} z+c_{3} x z+c_{4} x^{2}+c_{5} z^{2}
$$

and the functional $\Lambda$ is formed as

$$
\Lambda=\sum \sum(f-\hat{f})^{2},
$$

which is minimized to derive the coefficients of (27) that are used to calculate the first- and second-order spatial derivatives of $f$. Again, an area of $7 \times 7$ pixels was used in the application of (28). This method of estimating spatial derivatives employs some degree of filtering, which reduces the need for large areal coverage in the application of the Gaussian spatial filter.

\section{c. Validation tests}

Two error estimates were used to assess the accuracy of the wind calculations: a consistency test and a test using tracer beads in a kinematic flow field. The consistency test employed the assumption that the IR field moves with the flow, which is the basic assumption in the image flow analysis. For the assumption to be valid, it should be necessary to be able simply to translate $f$ at $t=t-\Delta t$ to positions at $t=t+\Delta t$ using the estimated wind components. The translated IR field then should be close to the observed IR field at $t=t+\Delta t$. The consistency error estimate for the winds $e_{c}$ is

$$
e_{c}=\frac{\int\left(f^{*}-f^{t-\Delta t}\right)^{2} d x d z}{\int\left(f^{t+\Delta t}-f^{t-\Delta t}\right)^{2} d x d z}
$$

where

$$
f^{*}(x, z)=f^{t+\Delta t}\left(x_{0}+2 d t u, z_{0}+2 d t w\right),
$$

and $x_{0}$ and $z_{0}$ are the grid positions at $t=t-\Delta t$. Only grid points where data actually were processed were considered in the application of (29) and (30). This approach can bias the error estimate to small values when the number of points analyzed is small due to high threshold standards, so the percentage of the area analyzed also must be kept in mind. The choice of the denominator in (29) was somewhat arbitrary, as would be instead using the number of data points, but the goal was to look for relative accuracy; if there are no gradients present, then (29) does not produce a false error estimate that indicates high accuracy. This error estimate was used extensively in tuning the spatial and temporal filters.

Obtaining low values of $e_{c}$ is only a necessary condition for the algorithm to have acceptable accuracy. The velocity component normal to the gradient of $f$ contributes by far the most to the achievement of a low error using this test. For example, in "gradient motion," such as the flow that might be predicted using Horn and Schunck (1981), little consideration is given to crossgradient flow. In visual imagery this lack is understandable but in fluid dynamics one expects to find significant cross-gradient flow, particularly in regions of vorticity production. In such cases, gradient motion may give low $e_{c}$ estimates even when the solution physically is unreasonable. Thus, this test can only be viewed as a necessary self-consistency condition.

The second test consisted of using an illustrative field of widely separated beads in kinematic flow fields. This test highlighted temporal aliasing effects as well as the performance of the image flow algorithm for small finite-sized structures in the flow. For a given temporal resolution, this type of test tends to overestimate difficulties in analysis because of the poorly defined gradients as compared to realistic thermodynamic fields.

Figure 3 shows a composite of beads in four different kinematic flow fields. The lower right and lower left are pure divergence and rotation, respectively, while the upper two flows are pure translation. The beads appear as local bright spots. Every third wind vector is plotted, showing the nature of the derived winds. In the case of 


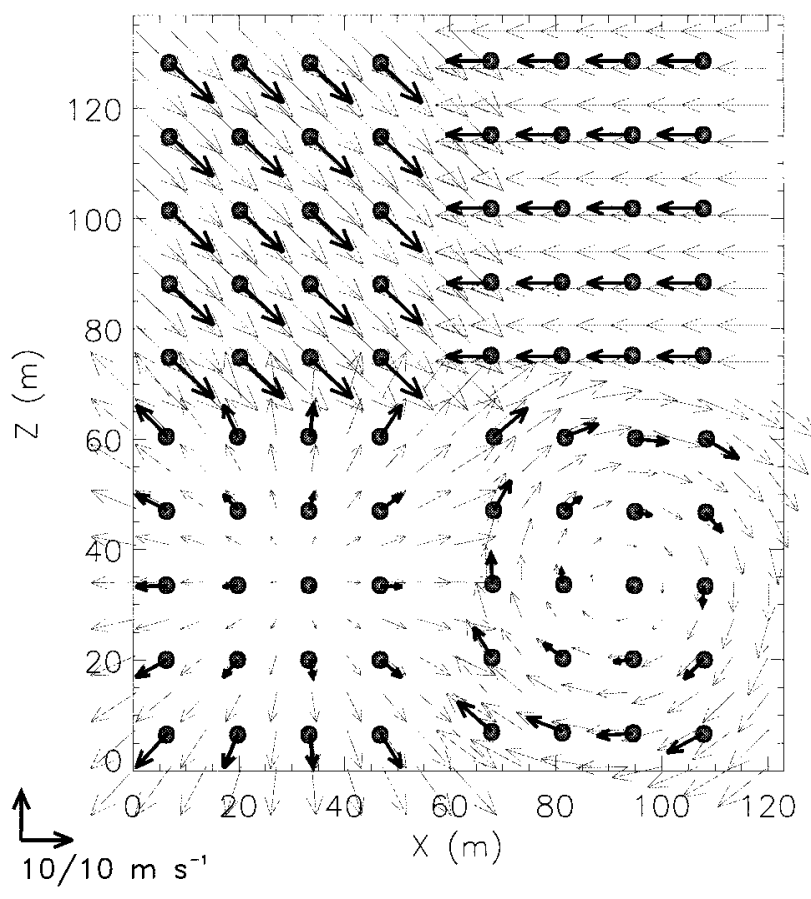

FIG. 3. Image flow for beads in prescribed kinematic flow fields. The flows are pure divergence, pure rotation, and two cases of pure translation. Both the analytical (light arrows) and image flow (dark thick arrows) vectors are plotted. Every third vector is shown for the case where the pixel motion was limited to one pixel per time frame. The derived vectors were averaged locally to give a single representation per bead.

Fig. 3 the estimated winds represent average values taken over the region of a bead, giving a single solution per bead. Comparison with results based on no local averaging showed that averaging tended to reduce accuracy slightly since the temporal resolution was relatively high; that is, the beads were allowed to move at most 1 pixel per time frame. The effect of allowing the beads to move a maximum of 4 pixels per time frame also was tested. In this case the wind vector solutions fan out, reducing accuracy significantly. Table 1 shows both the $e_{c}$ error estimates and the equivalent error for the velocity for a range of cases. The table shows errors ranging from 0.030 and 0.027 to 0.81 and 0.45 for the irradiance variance and velocity variance errors, respectively. The averaging of the vectors decreases the accuracy of the error variances to 0.089 and 0.027 for the $\Delta_{r}=1$ and $N_{g}=9$ case, as defined in Table 1 .

The results shown in Fig. 3 used $9 \times 9$ points in the Gaussian filter. Tests were also performed using $5 \times 5$ $\left(N_{g}=5\right)$ and $7 \times 7$ points. Here $e_{c}$ decreased significantly with increasing areal extent of the Gaussian filter. The errors at $N_{g}=5$ were 0.25 compared with 0.03 at $N_{g}=9$. The velocity variance errors (which can be calculated for this case) decreased in concert with the irradiance errors, indicating that the increase in accuracy was not simply a false reflection of increases caused by smoother fields.
TABLE 1. Error statistics for widely spaced beads in a kinematic field. Here $\Delta_{r}$ refers to maximum pixel motion per time step, $N_{g}$ is the order of the Gaussian filter, $e_{c}(T)$ is the variance error for the grayscale intensity, and $e_{c}\left(q^{2}\right)$ is the equivalent error for the squared velocity. All cases shown used the threshold velocity of $40 \mathrm{~m} \mathrm{~s}^{-1}$. "Avg" refers to whether a single estimate was derived through local averaging.

\begin{tabular}{ccccc}
\hline \hline$\Delta_{\mathrm{r}}$ & $N_{g}$ & $e_{c}(T)$ & $e_{c}\left(q^{2}\right)$ & Avg \\
\hline 1 & 5 & 0.306 & 0.087 & Yes \\
1 & 7 & 0.155 & 0.045 & Yes \\
1 & 9 & 0.089 & 0.027 & Yes \\
1 & 5 & 0.155 & 0.106 & No \\
1 & 7 & 0.099 & 0.075 & No \\
1 & 9 & 0.030 & 0.027 & No \\
2 & 9 & 0.207 & 0.106 & No \\
3 & 9 & 0.537 & 0.256 & No \\
4 & 9 & 0.814 & 0.451 & No \\
\hline
\end{tabular}

\section{Fire wind analyses}

The small errors obtained in the validation tests using beads could be considered only a necessary condition for the acceptance of the image flow algorithm. However, the fire wind estimates using (17) were unacceptably noisy, perhaps because of both the strong thermal gradients and the poor temporal resolution. The maximum translation is as much as 10 pixels per time frame in the IR imagery, which is well beyond any time resolution that we were able to handle accurately using (17) for the field of beads. However, the systematic structures in the IR fields offer an advantage compared to the beads, for which smoothly varying gradients had to be generated through filtering. Using (8) for the fire winds reduced the noise in the derived velocity $\mathbf{V}$ estimates to an acceptable level, allowing physical interpretation of the results. Note that the derived velocity components were highly consistent from frame to frame as seen in animations. (See the end of section 5 for a description of Web access to our animations.)

The IR images represent thermal data at the front of a fire line oriented at a mostly unknown and variable angle. The $x$ direction is along this front and $z$ is the vertical direction. Precise geometric positioning remains a problem to be resolved. At the end of the burn, the orientation was well known and was basically the southern boundary of the plot; many of the data shown in this paper are for this situation. In spite of the above limitations, the data irrefutably capture timescales and space scales. The consistency of the derived fields from frame to frame indicates that the spatial and temporal resolutions were adequate to observe the turbulent motions. As discussed earlier, the derived structure of the horizontal velocities indicates that the concentration of glowing soot was adequate to limit the depth to a shallow layer over which the IR camera took an average over the foremost flame surface. This phenomenon helps to explain the temperature range observed by the camera. Videos from the analyses show the frequent development of regions of saturation, suggesting forward 


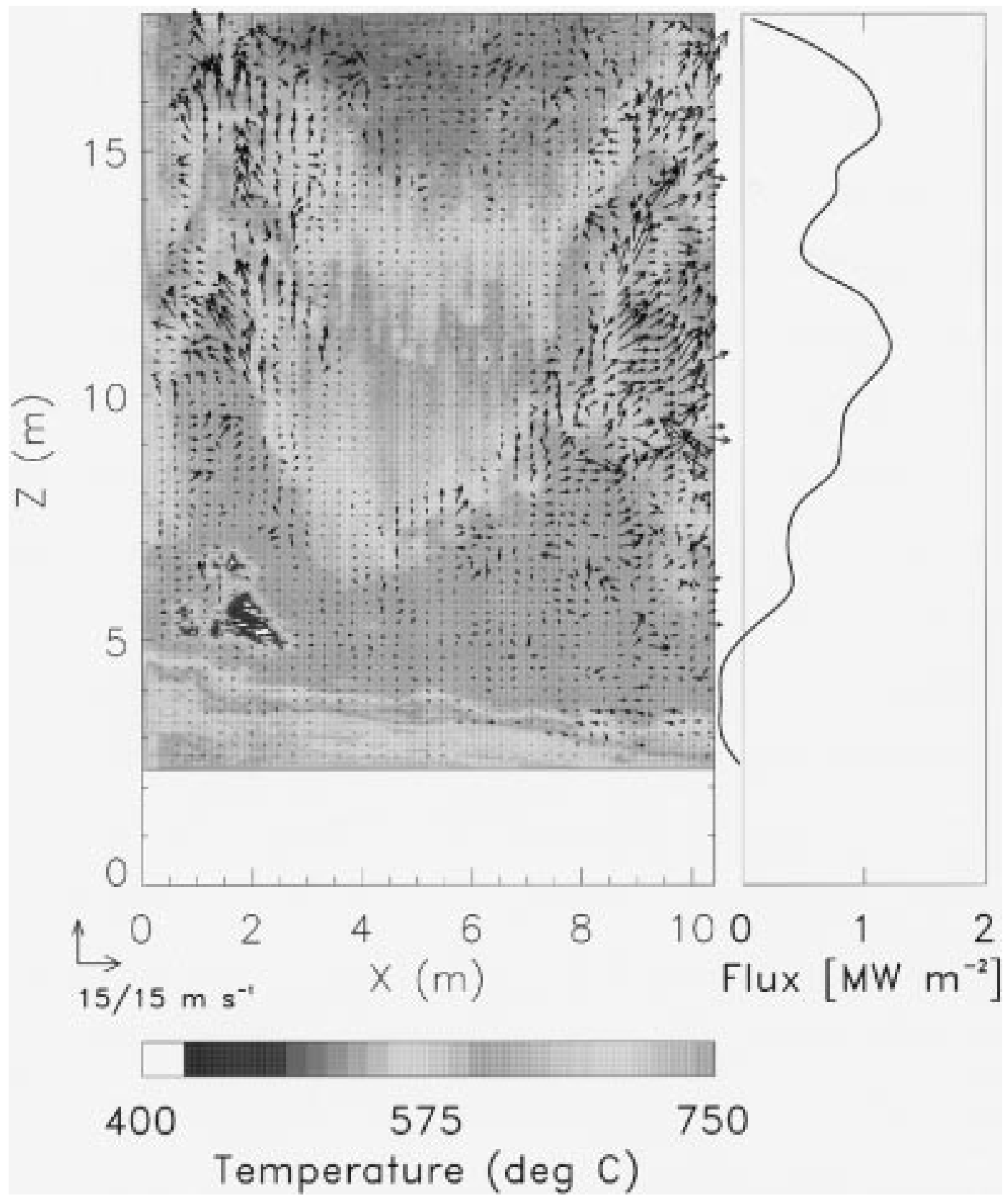

FIG. 4. Fire winds at $t=4 \mathrm{~min} 27 \mathrm{~s}$ into the plot- 6 burn. The first-order affine model for pure translation was solved using Eq. (8) and $7 \times 7$ pixels. The areal coverage of successful data analysis was $94.4 \%$ of the image. Three passes with the 1-2-1 time filter and a Gaussian filter of order 9 were applied to the raw data. (right) The estimated sensible heat flux $F_{s}$ in $\mathrm{MW} \mathrm{m}^{-2}$. No postfiltering was performed for this presentation.

bursts of thermals that bring the hotter combustion gases and flames into view.

\section{a. Derived estimates of horizontal and vertical rotation}

At $t=4 \min 27 \mathrm{~s}$, the plot- 6 burn was at its most intense, with processable data filling almost the entire image frame. We felt that this particular time was suf- ficiently demanding to provide a good evaluation of the analysis technique. Also, as mentioned earlier, the geometry of the image is easy to interpret at this time.

Figure 4 uses a reduced region of the full IR image and shows the estimated fire wind vectors overlying the background IR temperature field. The IR temperatures cover the full $400^{\circ}-750^{\circ} \mathrm{C}$ temperature range of the camera setting. For about the first $2 \mathrm{~m}$ above the ground the thermal field was saturated because the IR temper- 
TABLE 2. The $e_{c}$ statistics from the fire wind analyses. Here $e_{c}$ and $e_{c}$ (cond) refer to average values without and with the condition that $\Delta T<2^{\circ} \mathrm{C}$ was predicted. "Area" refers to percentage points of $e_{c}$; percentage points in the conditional sample are listed separately. Here NZ refers to the number of pixels in the vertical; there were 432 pixels in the horizontal. Wind components are in $\mathrm{m} \mathrm{s}^{-1}$.

\begin{tabular}{cccccccc}
\hline \hline Levels & Area & $\mathrm{NZ}$ & $e_{c}$ & $e_{c}$ (cond)/\% & $|u|_{\max } /|w|_{\max }$ & $t(s)$ \\
\hline 3 & 91.5 & 80 & 0.281 & $0.0115 / 60$ & 19.9 & 24.6 & $550 / 30 *$ \\
3 & 93.0 & 122 & 0.511 & $0.0335 / 60$ & 19.7 & 27.8 & $3050 / 30$ \\
3 & 94.2 & 142 & 0.364 & $0.0200 / 62$ & 15.2 & 19.6 & $4050 / 30$ \\
3 & 94.1 & 162 & 0.451 & $0.0241 / 50$ & 23.9 & 39.1 & $5050 / 30$ \\
3 & 96.0 & 246 & 0.681 & $0.0806 / 59$ & 15.9 & 17.1 & $6550 / 30$ \\
3 & 95.5 & 306 & 0.732 & $0.0544 / 46$ & 21.9 & 22.3 & $7050 / 30$ \\
3 & 92.2 & 414 & 0.882 & $0.0599 / 41$ & 30.7 & 29.1 & $8010 / 30$ \\
3 & 92.8 & 416 & 0.946 & $0.1093 / 41$ & 39.0 & 38.2 & $8020 / 30$ \\
3 & 94.3 & 418 & 0.653 & $0.0531 / 57$ & 22.7 & 22.1 & $8030 / 30$ \\
3 & 95.6 & 420 & 0.946 & $0.1030 / 58$ & 17.3 & 21.1 & $8050 / 30$ \\
3 & 95.6 & 420 & 0.824 & $0.0928 / 57$ & 15.6 & 25.0 & $8060 / 30$ \\
2 & 91.3 & 414 & 1.391 & $0.1540 / 64$ & 34.3 & 32.3 & $8010 / 30$ \\
2 & 92.8 & 416 & 1.191 & $0.2660 / 76$ & 23.1 & 29.1 & $8020 / 30$ \\
2 & 94.2 & 418 & 1.957 & $0.2860 / 74$ & 22.2 & 24.6 & $8030 / 30$ \\
2 & 94.8 & 420 & 1.669 & $0.2689 / 73$ & 17.7 & 24.4 & $8050 / 30$ \\
\hline
\end{tabular}

$*(=18.3 \mathrm{~s})$

ature exceeded $750^{\circ} \mathrm{C}$. These regions mostly are masked in the figure because of the effects of the filtering process. However, small regions remain visible near $x=$ $2 \mathrm{~m}$ and $z=7 \mathrm{~m}$. The maximum wind speeds shown are only $22 \mathrm{~m} \mathrm{~s}^{-1}$, which is well below the preoutlier and postoutlier threshold value of $40 \mathrm{~m} \mathrm{~s}^{-1}$. The analyses shown in Fig. 4 used the three-time-level approximation to derive the winds; that is, (8) and (24) were applied. When the two-time-level approximation was used by applying (25) instead of (24), a much noisier field was obtained, with maximum speeds of $29 \mathrm{~m} \mathrm{~s}^{-1}$. The basic structures of $\mathbf{V}$ were similar but had larger amplitudes. Using (25) also resulted in some wind speeds that exceeded the $40 \mathrm{~m} \mathrm{~s}^{-1}$ threshold value. Even so, their occurrence did not strongly affect the analyses.

Amplitudes of $e_{c}$ were much larger for the fire winds than for the field of beads, as shown in Table 2. The poor time resolution of the data gave $e_{c} \approx 1$ overall. However, when the trajectories were categorized by accepting points where $\Delta T_{\mathrm{IR}}$ was less than $2^{\circ} \mathrm{C}$, about half the data points had $e_{c}$ between 0.03 and 0.1 . The effect of using (25) was to increase $e_{c}$ significantly to approximately 2 . The conditional values $\left(\Delta T_{\mathrm{IR}}<2\right)$ increased to between 0.15 and 0.30 . The difference in accuracy resulting from using (24) versus (25) is most likely a result of data interlacing. In the future, we will attempt to correct for interlacing in our analysis. For this paper, only results using the three-time-level algorithm are shown.

Two rising towers of hot, rotating air are shown in Fig. 4. Wind vectors near the top of the tower on the right point upward and to the right, whereas the vectors near the top of the left tower point upward and generally toward the left. The systematic horizontal motion must result from rotation since the towers remain vertical with time. The systematic horizontal motion also suggests that the camera was viewing a shallow layer of soot in the forefront rather than seeing an average through a large portion of the tower where horizontal motions of opposite sign would occur. The apparent rotation is consistent with vorticity at the fire front undergoing enhanced vortex tilting. In this particular case the vortex on the left would result from tilting of a vortex on the left that was broken apart effectively by the enhanced buoyant acceleration, allowing the lower portion of the tilted vortex to maintain its rotational integrity. Similarly, the vortex on the right may result from similar enhanced vortex tilting on the right. If one estimates the amplitude of the vertical vorticity as the radial velocity divided by the radius of the rising tower, one obtains $\zeta$ $\approx \pm 4-10 \mathrm{~s} \mathrm{~s}^{-1}$, using radial velocities of $12-20 \mathrm{~m} \mathrm{~s}^{-1}$ and tower radii of $2-3 \mathrm{~m}$. These values are plausible for vertical vorticity and are approximately twice the magnitude reported in the simulations of Clark et al. (1996b). Events similar to this one occurred throughout the data analysis, pointing to the strong three-dimensional character of the fire winds.

Another aspect of the winds shown in Fig. 4 is that vortex tilting occurred in such a way that the two convective towers rotated in a sense that should have caused winds in the 6-m gap between towers to blow toward the camera, which is the general direction of fire propagation. This particular type of vortex tilting could be fundamental to the physics of fire propagation. However, these forward-bursting and relatively cool winds should not be confused with the hairpin vortices that can cause strong forward bursts of hot gases. Using the CFS IR line scanner, T. Blake (1997, personal communication) observed fingers of hot gases bursting forward near canopy height. These fingers probably are associated with the rising and forward-bursting motion (turbulent burst) associated with vortex tilting that eventually leads to the tilted vortex developing the hairpin shape. These shapes, we believe, are seen in our data 
as the rising saturated regions that the CFS was able to capture by viewing the fire from the side. The IR saturation prevents analysis of these regions. Clear evidence of these forward bursts also is seen in the USFS visual video taken from the same tower position. Unfortunately, smoke frequently obscures convective details, allowing only intermittent cases of forward bursting hairpin vortices to be observed.

The panel on the right of Fig. 4 shows the estimated sensible heat flux derived from the analyzed field of $w$ and the perturbation $T_{\mathrm{IR}}$. The heat flux $F_{s}$ was calculated as

$$
F_{s}=\rho c_{p}\left[w\left(T_{\mathrm{IR}}-\bar{T}_{\mathrm{IR}}\right)\right],
$$

where $\bar{T}_{\text {IR }}$ is the background IR temperature, $\rho$ is density, and $c_{p}$ is specific heat capacity. The use of (31) assumes that $\epsilon^{1 / 4} \approx 1$, where $\epsilon$ is the emissivity, allowing one to equate the air and IR temperatures. This assumption is a reasonable estimate for the intense crown fire but probably less appropriate for ground or grass fires. In any case, the approximations employed should result in an underestimate of $F_{s}$. A vertical 1-2-1 filter was applied to $F_{s}$ to provide a smooth vertical profile. The vertical structure of $F_{s}$ shows a gradual increase with height to about $12 \mathrm{~m} \mathrm{AGL}$, which is the approximate canopy height. The linear increase of $F_{s}$ with height is primarily a result of the vertical profile of available fuel. However, other aspects may contribute to this type of structure to some degree.

The uniform region of hot gas near the surface did not define $T_{\mathrm{IR}}$ sufficiently enough to allow the image flow analysis accurately to estimate $w$ near the surface, thus preventing estimates of $F_{s}$ in this region. This region also was where $T_{\mathrm{IR}}$ saturation most frequently occurred. The horizontal vortices near the surface at the fire front would rotate in the sense of rising motion over the fire with downdrafts in front and behind resulting from the return flow. Such flow structures could contribute directly to fire spread by drying and heating or even igniting by transport the fuel at the leading edge of the fire. Whereas the vortex tilting mechanism is inherently three-dimensional, this latter one is not and could be replicated in two-dimensional models such as those of Heilman and Fast (1992) and Linn (1997). Since the fire is driven by the $17 \mathrm{~km} \mathrm{~h}^{-1}$ mean flow wind, one might expect more compensating downdrafts to occur in front than behind. As shown in Fig. 5 by the contours of $|w|=2 \mathrm{~m} \mathrm{~s}^{-1}$, there is an almost equal distribution of downdraft and updraft air below $z \approx 8$ $\mathrm{m}$, suggesting a strong influence due to saturation.

\section{b. Wind component analysis}

Figure 5 shows four closely spaced time levels of $w$ contoured over the background IR temperature field. These plots are spaced only $0.1 \mathrm{~s}$ apart and show the rapidity with which significant changes occur in the fire. Figure 5 a represents the same time as Fig. 4 but shows the full width of the image. The structure and location of the updrafts are consistent with the apparent rising motion of the towers that one might visually estimate. However, the areal coverage of updrafts is somewhat fragmented. This fragmentation could be a result of either deficiencies in the image flow algorithm or could be caused by severe levels of turbulence. Regions of negative $w$ are evident throughout, although those regions closest to the surface are affected strongly by the IR image saturation.

Figure 6 shows the same four times as Fig. 5 but depicts $u$. The two rising towers on the left of the image show well-defined structures of $u$ consistent with the rotation described earlier. Both towers appear to rise almost vertically yet have well-defined regions where $|u| \approx 6 \mathrm{~m} \mathrm{~s}^{-1}$. Note that both $w$ and $u$ shown in Figs. 5 and 6 were postfiltered for presentation. In the cooler region just above the fire $(z>15 \mathrm{~m})$ a number of vertically elongated structures of alternating sign in $u$ are seen. Again these structures appear to be associated with rotating convective updrafts. It is interesting that structures of $u$ are at least as well defined in the image analysis as are structures in $w$. This finding most likely is caused by the ubiquity of rotation in the fire combined with a shallow thermal sampling by the IR camera.

Figure 7 shows a sequence of $T_{\mathrm{IR}}$ and wind vectors in the image plane $\mathbf{V}$ for four times separated by $66 \mathrm{~s}$. This figure shows a much longer period of the fire than was examined previously. The approaching fire can be seen by the lowering of both the top and bottom positions of the images. The horizontal (vertical) pixel sizes decrease substantially between Figs. $7 \mathrm{a}$ and $7 \mathrm{~d}$ from $0.15(0.12)$ to $0.059(0.049) \mathrm{m}$. Since the $x$ axis is changing with time, in both Figs. $7 \mathrm{c}$ and $7 \mathrm{~d}$ it is approximate and is taken from Fig. 7d. The figure shows that the regions of intense convective activity varied substantially with time. The amplitude of the updrafts appeared to be relatively uniform from one frame to the next when viewed as animations. The animations showed strong levels of variability over fractions of a second but structures were tracked easily from frame to frame. The flow is highly turbulent and, as such, each panel of Fig. 7 is only a snapshot of a quickly changing structure. One really has to view animations to appreciate better the data and the quality of the derived wind fields.

Animations of the data were developed using FORTRAN software; the figures in this paper were generated using IDL software. [An audio/video interleave (AVI) format animation could be viewed at the time of publication at http://www.mmm.ucar.edu/science/fire/ir/ ir1.html.] The animations of the plot-6 data show contours of the IR temperature overlaid with the derived wind vectors. The contours make it easier to discern the regions of saturation that periodically develop and can be followed easily as they propagate (probably forward) vertically out of the image domain. The Web page referenced above contains a visual from the USFS video 


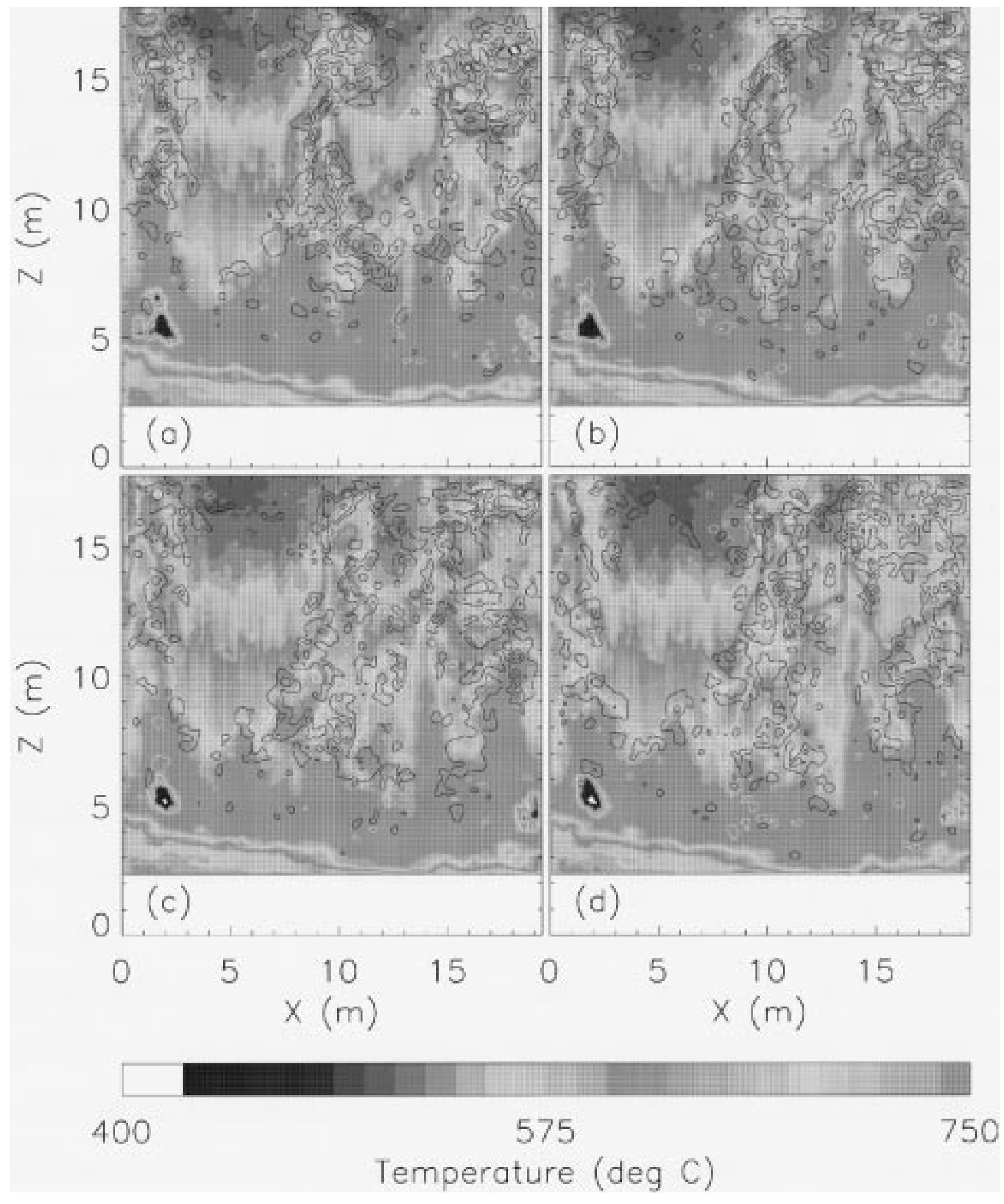

FIG. 5. Time sequence of $w$ overlying the IR temperature field for the full image. (a) The time shown is $267.167 \mathrm{~s}$; times for (b)-(d) are $0.1,0.2$, and $0.3 \mathrm{~s}$ later, respectively. Solid dark contours show $w$ values of 2,6 , and $10 \mathrm{~m} \mathrm{~s}^{-1}$. Solid white contours show $w$ values of -2 , -6 , and $-10 \mathrm{~m} \mathrm{~s}^{-1}$. Here $w$ was postfiltered for presentation. 


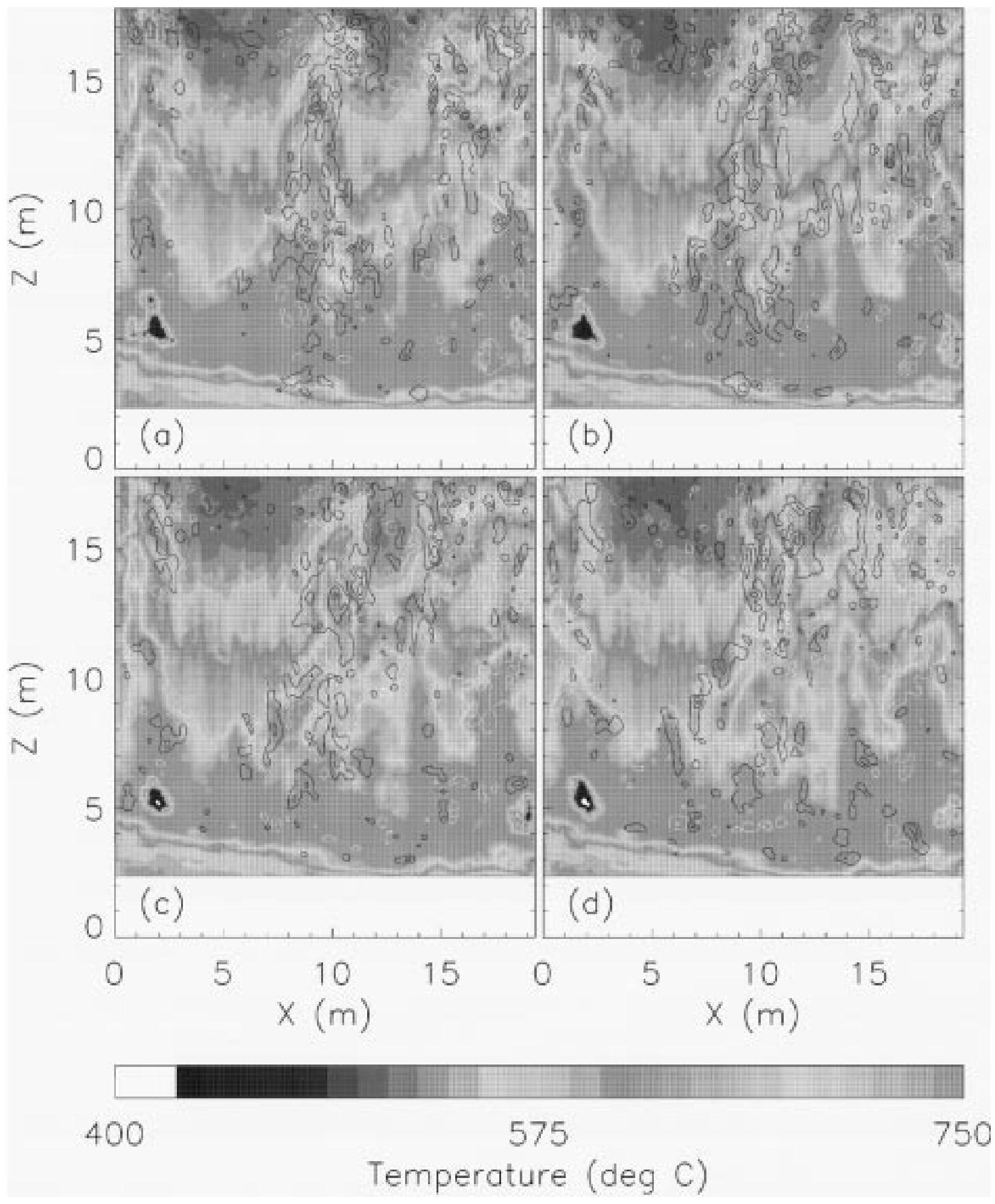

FIG. 6. Same as Fig. 5 but showing $u$. Solid dark contours show 2, 6, and $10 \mathrm{~m} \mathrm{~s}^{-1}$; solid white $-2,-6$, and $-10 \mathrm{~m} \mathrm{~s}^{-1}$. Here $u$ was postfiltered for presentation. 


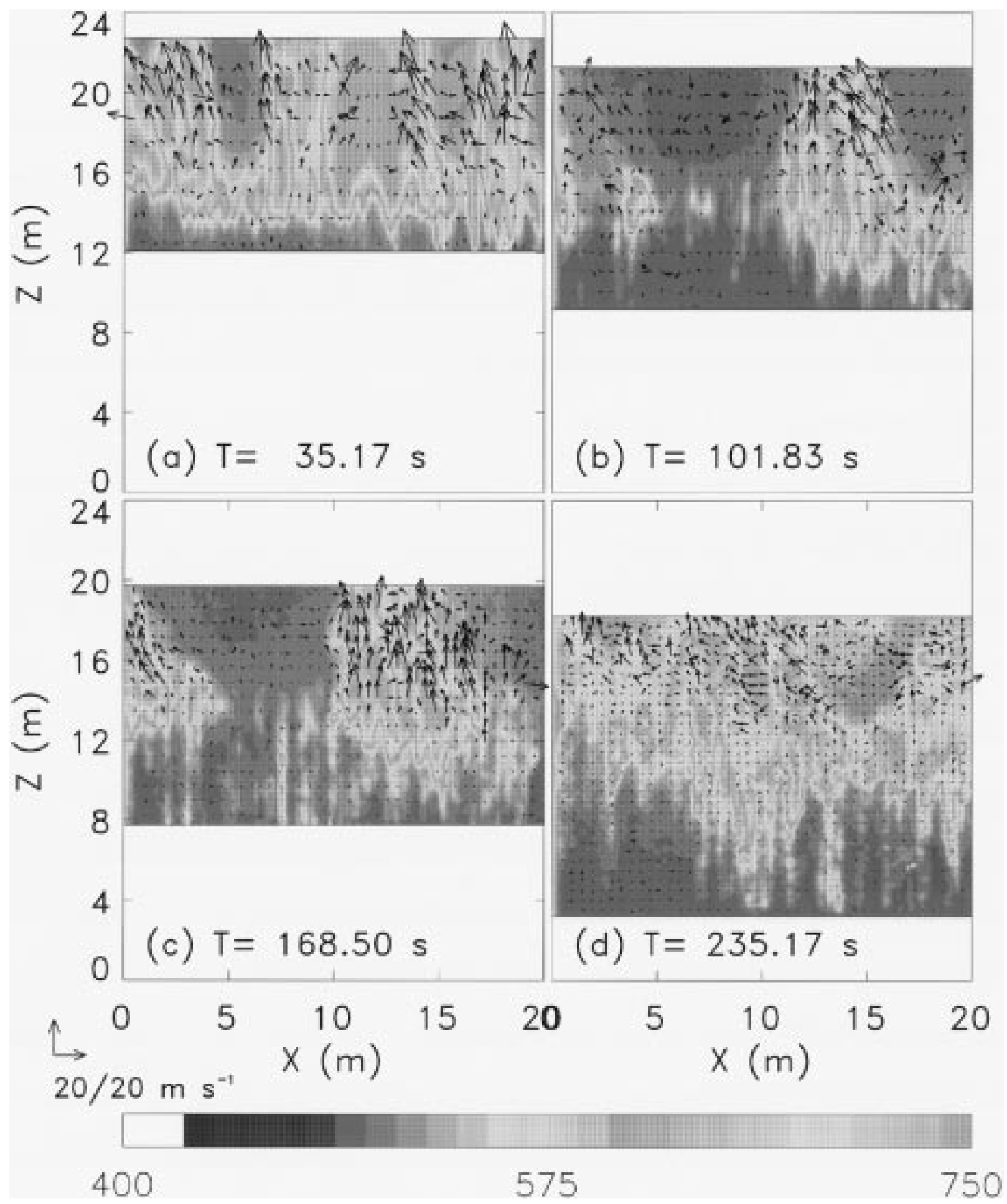

FIG. 7. A sequence showing $T_{\mathrm{IR}}$ and the derived wind vectors for four times spaced $66 \mathrm{~s}$ apart during the plot- 6 burn. 


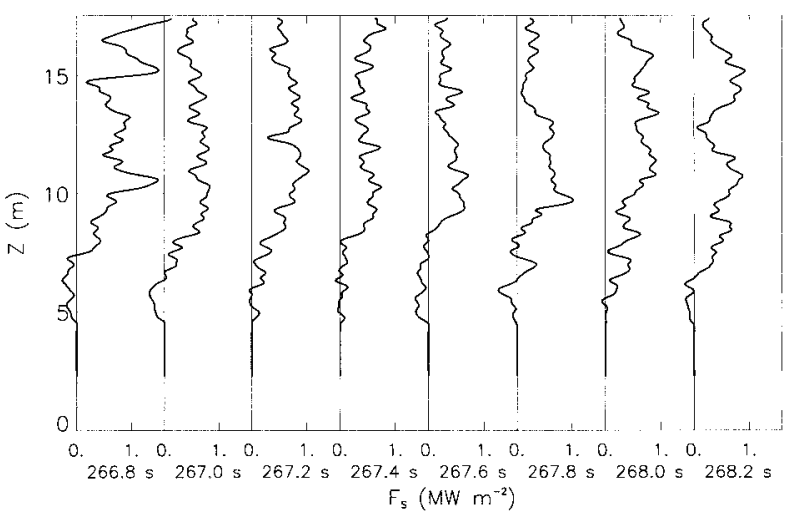

FIG. 8. Time sequence of vertical profiles of $F_{s}$ derived through image processing. Times are closely spaced from near the end of the plot-6 burn.

camera located on the same tower as the IR camera. It nicely captures a forward-bursting hairpin vortex.

\section{Fire wind statistics}

Images were analyzed at fixed intervals throughout the 4.5-min duration of the plot- 6 burn to calculate basic fire statistics and to assess the integrity or persistence of the analysis technique. Sixteen consecutive frames of $T_{\mathrm{IR}}$ were retained every $16.667 \mathrm{~s}$, allowing derivation of only eight consecutive image flow frames. At the last time level we retained 100 consecutive images, of which Fig. 4 was the first.

\section{a. Sensible heat flux}

Figure 8 shows eight vertical profiles of $F_{s}$ derived from the image analysis using (31). The profiles are spaced $0.2 \mathrm{~s}$ apart and are representative of all frames analyzed near this time. A linear increase of $F_{s}$ with height to $z \approx 13-15 \mathrm{~m}$ persists throughout. Maximum values range between 1.0 and $1.2 \mathrm{MW} \mathrm{m}^{-2}$ with means closer to 0.6 . Here $F_{s} \approx 0.5 \mathrm{MW} \mathrm{m}^{-2}$ physically is reasonable and could be obtained by burning, say, $2 \mathrm{~kg}$ $\mathrm{m}^{-2}$ or fine fuel (i.e., dead fuel less than $0.64 \mathrm{~cm}$ in diameter) in approximately $70 \mathrm{~s}$.

Figure 9 shows a sequence of 16 profiles of $F_{s}$ derived throughout the 4.5-min duration of the burn. The continual decrease in height of the flux profiles with time results from the reduced FOV as the fire approaches the camera, and the abrupt end at the top of the profiles represents the end of the image data. Only when the fire breaks through the trees bordering the southern clearing (the last frame) can we see the full vertical extent of the heat flux from just above the ground to the convection above canopy height. The amplitude of $F_{s}$ shows a general trend of decreasing substantially with time. This trend is particularly true for early profiles where $F_{s}$ has amplitudes exceeding $3 \mathrm{MW} \mathrm{m}^{-2}$. Miscalculations of $\Delta z$ for these early times would have to be in

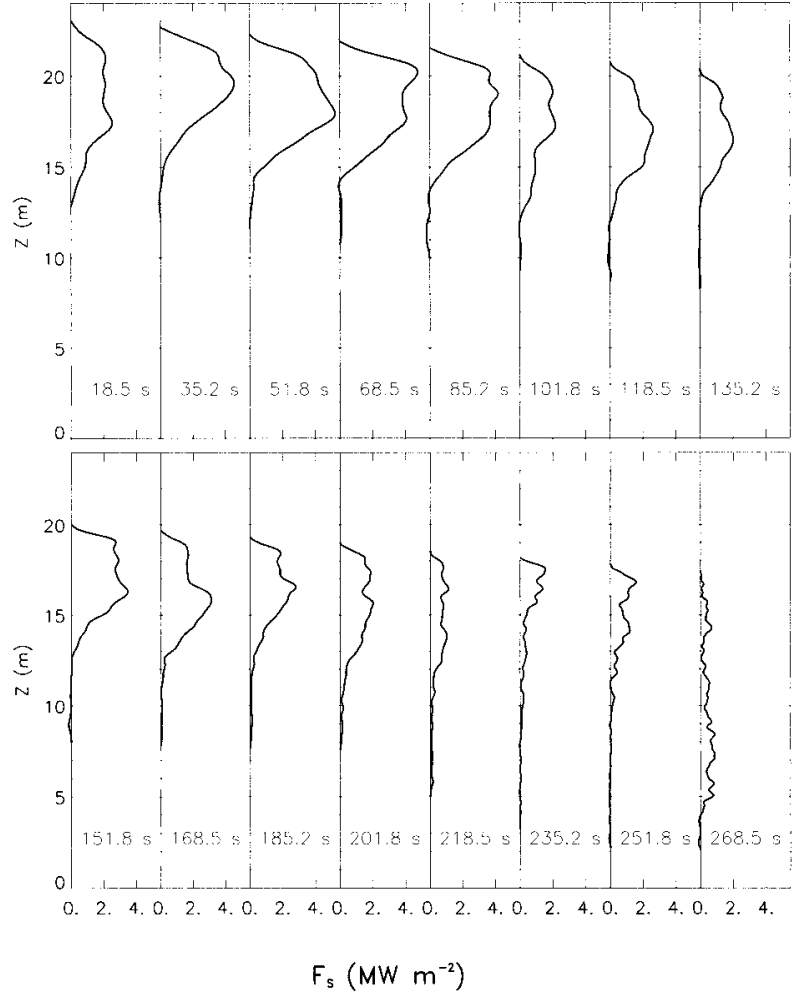

FIG. 9. Same as Fig. 8 but times are spaced equally throughout the 4.5-min duration of the plot-6 burn.

error by a factor of almost 3 to account for this trend. Since the amplitude of $w$ from image analysis scales proportionally to $\Delta z / \Delta t$ (for a fixed $\Delta x / \Delta z$ ratio), an overestimation of $\Delta z$ overestimates $w$ and therefore $F_{s}$. This type of error seems unlikely and would appear consistently throughout other statistics such as all components of the velocity extremes shown in Fig. 10. The large early maxima in $F_{s}$, on the other hand, could be a direct result of the time it takes convection to adjust to the presence of the fire, that is, temporary heat buildup just above the canopy, or to contributions from the napalm. Clark et al. (1996a,b) showed in their time history of buoyancy and velocity extremes that it took about 1-2 min for the local convection to adjust to the local thermal forcing before a more efficient convection of heat away from the fire could organize. Further assessment of this issue will have to wait for evaluation through modeling studies.

\section{b. Wind extremes}

Figures 10a and 10b show time histories of $u$ and $w$ extremes throughout the plot- 6 burn. Here $\mathbf{V}$ was calculated at eight consecutive times spaced $0.033 \mathrm{~s}$ apart at intervals of $16.67 \mathrm{~s}$ from $t=16.67 \mathrm{~s}$ to $t=285.00$ s, giving only 128 samples in time. To assess the statistical nature of the data, extremes were plotted using evaluations of means and standard deviations. With 

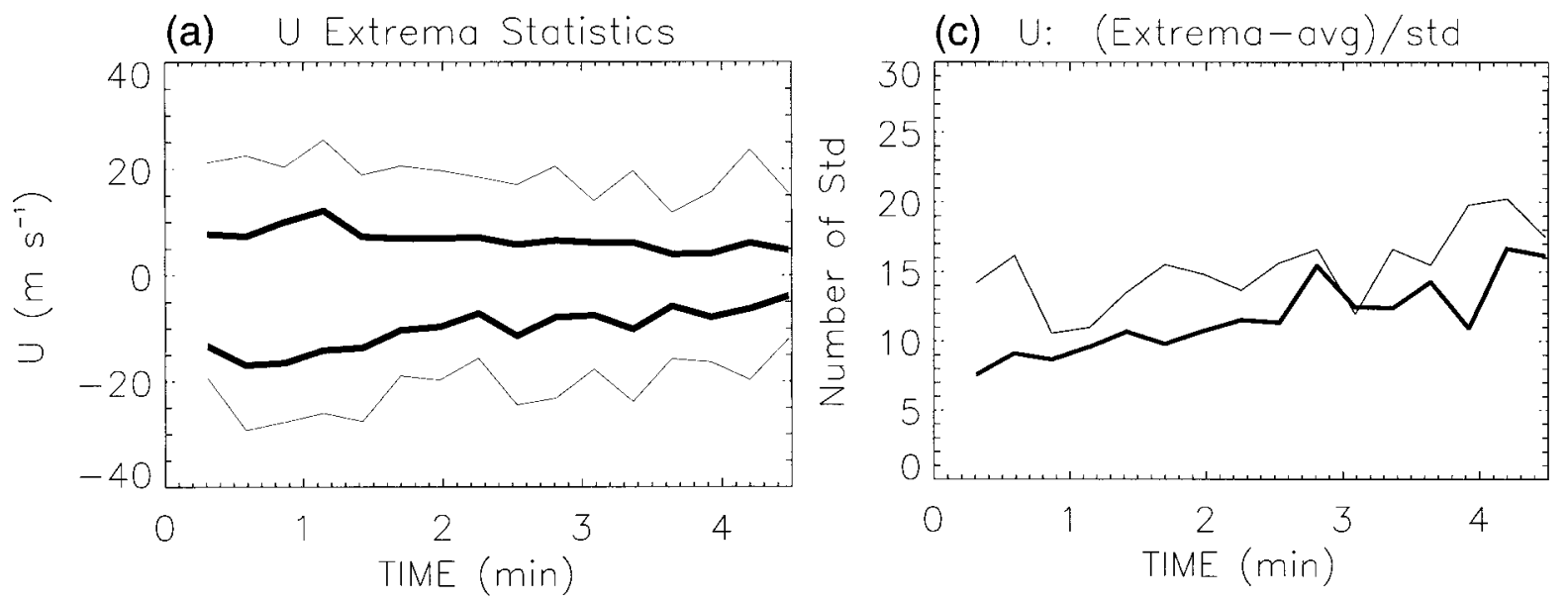

(b) W Extrema Statistics
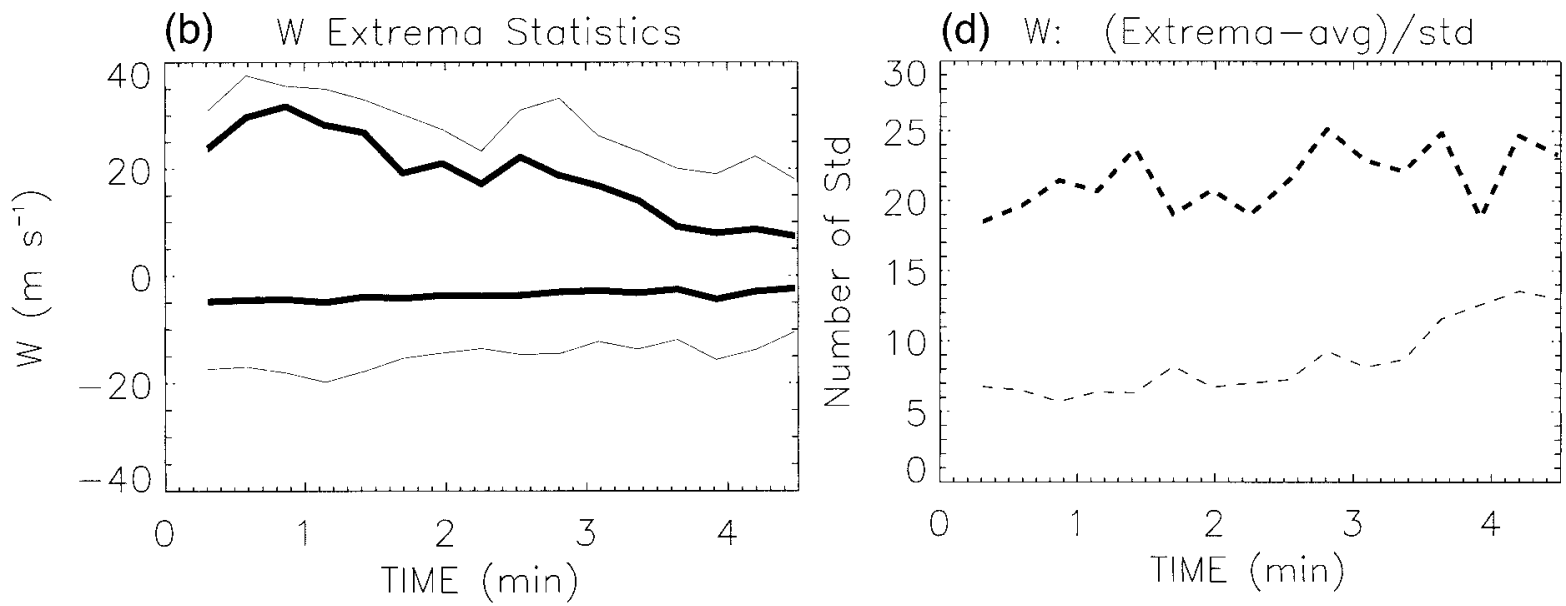

FIG. 10. Time series of derived wind component extremes. (a) The thin lines show $u_{\max } / u_{\min }$ and the thick lines show $\left\langle u_{+}\right\rangle+5 \sigma_{u_{+}}$and $\left\langle u_{-}\right\rangle-5 \sigma_{u_{-}}$, where \langle\rangle indicates a mean quantity and the \pm subscripts refer to positive or negative velocity components; (b) similar to (a) but for $w_{\max } / w_{\min }$ and $\left\langle w_{+}\right\rangle+5 \sigma_{w_{+}}$and $\left\langle w_{-}\right\rangle-5 \sigma_{w_{-}}$; (c) time history of the number of standard deviations by which the $u$ velocity extrema deviate from their means-thick is for negative and thin for positive $u$; (d) similar to (c) but for $w$. Each of the 15 data points shown was derived by postaveraging the estimates of the point extremes and standard deviations over the eight available consecutive frames.

Gaussian- or normally distributed data, extremes would occur at approximately five standard deviations $(\sigma)$ from the mean. Large deviations of extremes beyond their 5- $\sigma$ estimates could suggest that either the data are nonGaussian or that the extremes might be influenced by artifacts of the image flow algorithm. The heavy lines in Figs. 10a and 10b show the statistical estimates of $u$ and $w$ extremes, plotted as $u_{\max / \min }=\left\langle u_{ \pm}\right\rangle \pm 5 \sigma_{ \pm}$, where \langle\rangle indicates the mean. Updrafts were categorized separately from downdrafts; the \pm subscript means that only positive or negative field values were analyzed. There is a well-defined trend in the 5- $\sigma$ statistical estimates of maximum $w$. It decreases from almost $30 \mathrm{~m}$ $\mathrm{s}^{-1}$ at about $0.5 \mathrm{~min}$ to below $10 \mathrm{~m} \mathrm{~s}^{-1}$ at $3.5 \mathrm{~min}$. This trend corresponds to the same variation we saw in the estimates of $F_{s}$. The comparison for negative $w$ is quite different, where the point values maintain about -20 $\mathrm{m} \mathrm{s}^{-1}$ throughout and the 5- $\sigma$ estimate shows an almost constant $-5 \mathrm{~m} \mathrm{~s}^{-1}$. There is also a trend toward decreasing amplitudes with time for $u$ but it is quite minor in comparison with that seen for positive $w$. If the point estimates of the extremes (thin lines) result mainly from artifacts such as thresholding in the image processing, then one might expect a reasonably erratic or large separation between them and their 5- $\sigma$ estimates.

Figures 10c and 10d show the time history of the number of standard deviations by which the velocity extremes deviate from their means. The figures show that $w_{\max }$ is initially near 6 standard deviations and systematically increases to 13 standard deviations from its mean. On the other hand, $w_{\min }$ is consistently about 20 standard deviations from its mean for the entire burn. The statistics for $u$ show that both positive and negative value extremes range between 10 and 15 standard deviations from their means. These results suggest that the derived velocity extremes most likely are resulting from artifacts of the analysis scheme, or the distribution is non-Gaussian. The values of $w_{\max }$ for the first 2-3 min of the burn may represent an exception. The problems, if such is the explanation, appear to be most severe for 


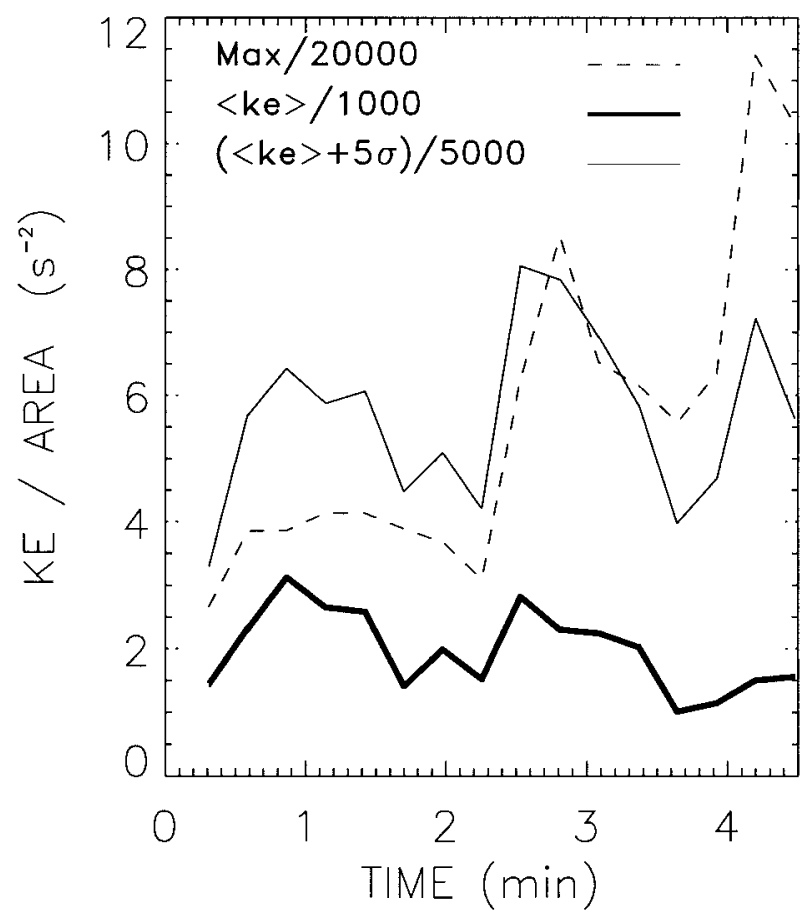

FIG. 11. Same as Fig. 10 but for the kinetic energy statistics. Each of the 15 data points shown was derived by postaveraging the estimates of $\mathrm{ke}_{\max },\langle\mathrm{ke}\rangle$, and $\sigma_{\mathrm{ke}}$ over the eight available consecutive frames.

downdrafts. Considering these results, we feel that the $u$ and $w$ extreme estimates derived using statistical moments and, say, 5- $\sigma$ deviations, are probably more meaningful than are point estimates.

In further developments of the image flow algorithm this type of statistical comparison of $u$ and $w$ extreme estimates will be evaluated to see if the point and statistical moment estimates can be made to compare more favorably. A convergence of the point and, say, 5- to 7- $\sigma$ estimates, along with improved $e_{c}$ errors, would greatly increase confidence in the details of the image flow analysis.

\section{c. Kinetic energy}

Figure 11 shows a time history of kinetic energy statistics, including the point maximum, statistical moment estimate of the maximum assuming a 5- $\sigma$ deviation, and the spatial average. Each of the 15 points on the plot is composed of averages over the eight consecutive frames analyzed for each window of data. Each point represents an average over $0.267 \mathrm{~s}$. These results basically were insensitive to this local averaging in time. There is an oscillation with a period of approximately 2 min that is apparent in all representations of kinetic energy in the figure. We see two maxima at $t=0.75$ and $2.75 \mathrm{~min}$ and two minima at $t=1.75$ and 3.75 min, suggesting that the fire intensity may be increasing and decaying over this period. There is surprisingly little detectable variation, other than this oscillation, in a mean trend with time. The plot shows averages per unit area observed and, as such, is probably the best that can be done to evaluate mean overall characteristics of the fire from the small sampling area. This is another characteristic that one might attempt to assess through modeling.

\section{Conclusions}

A gradient-based image flow analysis scheme was applied to the IR temperature observations of a prescribed crown fire in the Northwest Territories to derive fire winds and sensible heat flux. The results show that the technique is capable of deriving motions on a range of the small spatial and temporal scales associated with crown fire convection. We believe these scales of motion play an essential role in determining spread rates and some other aspects of fire behavior.

It is well known that strong rotation is present in fires. This fact was certainly true here where rotation was visually evident throughout the fire at the time of the burn. The derived motions show that vortex tilting occurs through a major portion of the observations and plays an integral role in the convective portion of fire behavior and heat transport. Vertical vorticity, or rotation about the vertical axis, was identified by the presence of strong horizontal motion in vertically rising towers. Rough estimates of vertical vorticity of between 4 and $10 \mathrm{~s}^{-1}$ were made using the amplitudes of the horizontal velocities and tower diameters. These vortices were embedded in updrafts of between 20 and $30 \mathrm{~m}$ $\mathrm{s}^{-1}$. In one example, the sense of the rotation was consistent with the tilting of horizontal vortices produced by the fire's horizontal thermal gradients.

The analyses pointed to a number of physical mechanisms that play active roles in determining the rate of fire spread. The image flow analysis was able to identify the tilting of vortices, leading to counterrotating convective towers. The sense of rotation in one example analyzed was shown to be in the sense that air between the towers would blow in the direction of fire spread at canopy height and below. Probably the most important vortex tilting evident in the data was that leading to what we believe are forward-bursting hairpin vortices. However, these vortices were unfortunately the least able to be analyzed using image flow techniques because the forward motion led to saturation of the IR image, that is, $T_{\mathrm{IR}}$ greater than $750^{\circ} \mathrm{C}$. The frequent occurrence of the rising and presumably forward-bursting regions of saturation were evident in the animation (see end of section 5 for details). Supporting evidence was discussed in the text. There was also some evidence that return flow downdrafts at the leading edge of the fire exist in the data. However, these regions usually were saturated. Further experiments using IR imagery should attempt to observe a broader range of IR temperature to capture both the cooler convection and the high-tem- 
perature regions in the forward-bursting vortices and in the near-surface fire.

Profiles of sensible heat flux were calculated by assuming that air temperature could be approximated using the IR temperatures. Maximum values of $F_{s}$ ranged between approximately 0.7 and $3 \mathrm{MW} \mathrm{m}^{-2}$ over the duration of the burn with the early time periods showing surprisingly large estimates of almost $3 \mathrm{MW} \mathrm{m}^{-2}$. Near the end of the burn, when the full vertical structure of the fire was visible to the IR camera, the profiles linearly increased to a magnitude compatible with fuel loads of about $2 \mathrm{~kg} \mathrm{~m}^{-2}$ and fine fuel burnout times of $70 \mathrm{~s}$.

Time histories of velocity extremes were compared with estimates by using statistical moments. With the exception of updrafts, the extremes were shown to depart significantly from estimates based on five standard deviations from their spatial mean. The departures for the downdrafts, in particular, and the $u$ extremes were beyond what one might expect even for non-Gaussian distributed data. For example, the downdraft extremes were approximately 20 standard deviations from their mean and the $u$ extremes were between 10 and 15 standard deviations away. This analysis suggests that the point estimates of the extremes in the motion field still are being influenced by artifacts of the image-processing scheme. The statistically determined extremes, using five standard deviations from the mean, are more likely to be representative at this time. They suggest that updrafts of between 10 and $30 \mathrm{~m} \mathrm{~s}^{-1}$, downdrafts of between -10 and $-20 \mathrm{~m} \mathrm{~s}^{-1}$, and horizontal motions of between 5 and $15 \mathrm{~m} \mathrm{~s}^{-1}$ frequently occurred throughout the fire.

Time histories of the mean and extreme values of area-weighted kinetic energy showed a strong oscillation in its strength. The data suggested there was a period of about $2 \mathrm{~min}$. Unfortunately, since the entire dataset is only $4.5 \mathrm{~min}$ long, this is too short for a reliable estimate. Larger crown fires of this intensity are not likely to be prescribed, but again, this type of dynamics could be evaluated further through modeling studies and, perhaps, in airborne remote sensing observations of wildfires.

In addition to statistics of the derived fire winds, two other measures were used to evaluate the accuracy of the image flow algorithm. Calculations from an idealized field of widely spaced beads in kinematic flow fields were used, where it was shown that image flow gave accurate results for good time resolution, that is, when the beads did not move more than 1 or 2 pixels per time frame. These tests were obtained using an algorithm that assumed that wind components were purely translational. A second measure used was a consistency error defined as the sum of squared differences between the advected field and its known future value. With good time resolution, normalized values between 0.02 and 0.05 were obtained for the field of beads. By comparing the predicted winds with their known values, we were able to show that $e_{c}$ also was representative of the accuracy of the wind predictions.

The $e_{c}$ errors were much larger for the fire winds where mean values of between 0.6 and 2.0 were obtained. When thresholding was used to reject points where the predicted temperature difference exceeded $2{ }^{\circ} \mathrm{C}$, much lower $e_{c}$ values between 0.03 and 0.1 were found over more than half of the processed data. This finding indicates that the large $e_{c}$ errors are not necessarily a good representation of the overall accuracy because exaggerated contributions occurred in regions of strong gradients where small errors in velocity predictions could disproportionately amplify the error estimates. Thus, alternate error estimates must be established to assess the image flow accuracy. The $e_{c}$ values were surprisingly sensitive to the numerical details of the image flow algorithm. For example, the seemingly paradoxical result was obtained that it was more accurate to use a lower temporal resolution scheme, that is, using three time levels spaced $0.067 \mathrm{~s}$ apart versus two spaced $0.033 \mathrm{~s}$ apart. The $e_{c}$ errors using three time levels were less than half those of the two-time-level scheme. The large $e_{c}$ errors for the fire winds probably result from the combination of extreme thermal gradients and rapid motion in the flow, which reduces the temporal resolution. The effect of variable temporal resolution also was demonstrated by the $e_{c}$ errors systematically increasing as the fire approached the near-plot boundary and the image pixel size decreased. Initially $e_{c}$ was about 0.3 , and it increased to about 1.0 near the end of the burn.

The current results also provide guidance in the design of future fire observations using IR imagery. For example, knowledge of the expected flow speeds can be used to match pixel size to frame rate in an attempt to optimize temporal resolution of the data when such possibilities exist. For example, the present observations probably were taken too close to the fire, resulting in overly small pixel sizes. Refined image analysis software may also allow derivation of divergence and vorticity fields that can be used to assess further details of the flow. Current estimates of divergence and vorticity (in the image plane) showed too much fine structure to allow much physical interpretation. Current plans for our future research in this area include using high-resolution model simulations to help to interpret the present data as well as to assess model physics. We also plan to enhance our IR observational database in the near future by including some prescribed fires in tropical grasses and intense forest fires of opportunity over the northwestern United States. It also was gratifying to find strong rotational motions throughout these tests. Not only do these observations confirm our fire model results, but, as expected, they point toward a powerful dynamic mechanism for violent fire behavior and rapid fire spread.

Perhaps the most important experimental task is to establish what it is that the IR camera is viewing. As 
discussed in the text, the coherency and consistency of the derived velocity components (particularly in the horizontal) suggest that the high emissivity of glowing soot is limiting the depth of view to a shallow layer at the flame front. A test of this hypothesis might be to perform experiments using both IR and visual cameras using either $\mathrm{CH}$ or $\mathrm{C}_{2}$ filters. The $\mathrm{CH}$ and $\mathrm{C}_{2}$ radicals occur only in the combustion zone and comparison between such IR and visuals should be informative.

These results are considered preliminary but overall they are both encouraging and exciting. They point to a number of areas where further research is required, such as image flow testing and development. Furthermore, some results remain interpretative and require further evaluation and validation through modeling studies. The derived fields and their subsequent analyses represent a starting point as a dynamic and thermodynamic dataset for model validation and understanding of forest fires.

Acknowledgments. We thank Laurie Gibson, David Suter, and Alireza Bab-Hadiashar for their helpful discussions on image analysis. We gratefully thank the staff at the Northwest Territories burns for their assistance collecting these data, particularly Bob Schuette, Paul Sopko, and Jack Cohen, who scaled the USFS tower to mount the camera. The assistance and advice of Dr. Brian Stocks of Forestry Canada were invaluable. We also thank the Mathematics Department at Monash University for the use of their local computing system, which was used to perform this analysis. One of the authors, J. Coen, is supported under USDA Forest Service Research Joint Venture Agreement INT-97034RJVA. Special thanks also to Inframetrics for their technical support and to Dr. Robert Serafin (NCAR director) whose support helped foster this line of research.

\section{REFERENCES}

Alexander, M. E., B. J. Stocks, B. M. Wotton, M. D. Flannigan, and J. B. Todd, 1998: The International Crown Fire Modelling Experiment: An overview and progress report. Preprints, Second Conf. on Fire and Forest Meteorology, Phoenix, AZ, Amer. Meteor. Soc., 20-23.

Bab-Hadiashar, A., and D. Suter, 1995: 2-D motion extraction using an image interpolation technique. SPIE-The International Society for Optical Engineering: Applications of Digital Image Processing XVIII, A. G. Tescher, Ed., SPIE, 271-281.

— , and - 1997: Optic flow calculation using robust statistics. Proc. Computer Vision and Pattern Recognition Meeting, Puerto Rico, IEEE, in press.

- - , and R. Jarvis, 1996: Investigation of the image-interpolation and differential optic flow techniques. Tech. Rep. MECSE 1996-1, Monash University, Australia, 31 pp. [Available from Department of Electrical and Computer Systems Engineering, Monash University, Clayton, Vic. 3168, Australia.]

Baines, P., 1990: Physical mechanisms for the propagation of surface fires. Math. Comput. Model., 13, 83-94.
Banta, R. M., L. D. Olivier, E. T. Holloway, R. A. Kropfli, B. W. Bartram, R. E. Cupp, and M. J. Post, 1992: Smoke-column observations from two forest fires using Doppler lidar and Doppler radar. J. Appl. Meteor., 31, 1328-1349.

Church, C. R., J. T. Snow, and J. Dessens, 1980: Intense atmospheric vortices associated with a $100 \mathrm{MW}$ fire. Bull. Amer. Meteor. Soc., 61, 682-694.

Clark, T. L., M.-A. Jenkins, J. Coen, and D. Packham, 1996a: A coupled atmosphere-fire model: Convective feedback on fireline dynamics. J. Appl. Meteor., 35, 875-901.

,,--- , and - , 1996b: A coupled atmosphere-fire model: Convective Froude number and dynamic fingering. Int. J. Wildland Fire, 6, 177-190.

Ditchburn, R. W., 1963: Light. Blackie and Son, Limited, 833 pp.

Grishin, A. M., 1992: Matematicheskoye modelirovaniye lesnykh pozharov i novyye sposoby bor'by s nimi (Mathematical Modeling of Forest Fires and New Methods of Fighting Them). Novosibirsk, $408 \mathrm{pp}$.

Heilman, W. E., 1992: Atmospheric simulations of extreme surface heating episodes on simple hills. Int. J. Wildland Fire, 2, 99114.

— development above lines of extreme surface heating. Int. J. Wildland Fire, 2, 55-68.

Hidalgo, L. G., 1993: Features of a smoke plume determined from successive photographs. Meteor. Mag., 122, 218-220.

Horn, B. K. P., and B. G. Schunck, 1981: Determining optical flow. Artif. Intell., 17, 185-204.

Linn, R., 1997: A transport model for prediction of wildfire behavior. Ph.D. dissertation, New Mexico State University, 195 pp.

McGrattan, K., H. R. Baum, and R. G. Rehm, 1996: Numerical simulation of smoke plumes from large oil fires. Atmos. Environ., 30 (24), 4125-4136.

McRae, D. J., and M. D. Flannigan, 1990: Development of large vortices on prescribed fires. Can. J. For. Res., 20, 1878-1887.

Nagel, H.-H., 1983: Displacement vectors derived from second-order intensity variations in image sequences. Comput. Vision, Graphics, Image Process., 21, 85-117.

Nobel, I. R., G. A. V. Bary, and A. M. Gill, 1980: McArthur's firedanger meters expressed as equations. Aust. J. Ecol., 5, 201203.

Radke, L. F., D. A. Hegg, J. D. Nance, P. V. Hobbs, K. K. Laursen, R. E. Weiss, P. J. Riggan, and D. E. Ward, 1991: Particulate and trace gas emissions from large biomass fires in North America. Global Biomass Burning, J. P. Levine, Ed., The MIT Press, 209224.

Rickel, C., B. Lamb, A. Guenther, and E. Allwine, 1990: An infrared method for plume rise visualization and measurement. Atmos. Environ., 24A, 2835-2838.

Rothermel, R. C., 1972: A mathematical model for predicting fire spread in wildland fuels. USDA Forest Service Res. Paper INT115, Intermountain Forest and Range Experiment Station, Ogden, UT, $40 \mathrm{pp}$.

Schunck, B., 1986: The image flow constraint equation. Comput. Vision, Graphics, Image Process., 35, 20-46.

Templeman, B., S. Raman, and S. Templeman, 1990: Analysis of vertical dispersion and relative concentration of an elevated plume in the tropical boundary layer using video digitization. Atmos. Environ., 24A, 853-858.

Tieszen, S. R., V. F. Nicolette, L. A. Gritzo, J. K. Holen, D. Murray, and J. L. Moya, 1996: Vortical structures in pool fires: Observation, speculation, and simulation. Rep. SAND96-2607, UC722, Sandia National Laboratories, Albuquerque, NM, 95 pp.

Tuttle, J. D., and G. B. Foote, 1990: Determination of the boundary layer airflow from a single Doppler radar. J. Atmos. Oceanic Technol., 7, 218-232.

Verri, A., F. Girosi, and V. Torre, 1990: Differential techniques for optical flow. J. Opt. Soc. Amer., 7A, 912-922. 